\title{
KELT-11b: A Highly Inflated Sub-Saturn Exoplanet Transiting the $V=8$ Subgiant HD 93396
}

Joshua Pepper ${ }^{1}$, Joseph E. Rodriguez ${ }^{2}$, Karen A. Collins ${ }^{2,3}$, John Asher Johnson ${ }^{4}$, Benjamin J. Fulton ${ }^{5}$, Andrew W. Howard ${ }^{5}$, Thomas G. Beatty ${ }^{6,7}$, Keivan G. Stassun ${ }^{2,3}$, Howard Isaacson ${ }^{8}$, Knicole D. Colón ${ }^{1,9,10,11}{ }^{\text {, Michael B. Lund }}{ }^{2}$, Rudolf B. Kuhn ${ }^{12}$, Robert J. Siverd ${ }^{13}$, B. Scott Gaudi ${ }^{14}$, T. G. Tan ${ }^{15}$, Ivan Curtis ${ }^{16}$, Christopher Stockdale ${ }^{17,18}$, Dimitri Mawet ${ }^{19,20}$,

Michael Bottom ${ }^{19,20}$, David James ${ }^{21}$, George Zhou ${ }^{4}$, Daniel Bayliss ${ }^{22}$, Phillip Cargile ${ }^{4}$, Allyson Bieryla ${ }^{4}$, Kaloyan Penev ${ }^{23}$, David W. Latham ${ }^{4}$, Jonathan Labadie-Bartz ${ }^{1}$, John Kielkopf ${ }^{24}$, Jason D. Eastman ${ }^{4}$, Thomas E. Oberst ${ }^{25}$, Eric L. N. Jensen ${ }^{26}$, Peter Nelson ${ }^{27}$, David H. Sliski ${ }^{28}$, Robert A. Wittenmyer ${ }^{29,30,31}$, Nate McCrady ${ }^{32}$, Jason T. Wright ${ }^{6,7}$, Howard M. Relles ${ }^{4}$, Daniel J. Stevens ${ }^{14}$, Michael D. Joner ${ }^{33}$, and Eric Hintz ${ }^{33}$

${ }^{1}$ Department of Physics, Lehigh University, 16 Memorial Drive East, Bethlehem, PA 18015, USA

2 Department of Physics and Astronomy, Vanderbilt University, 6301 Stevenson Center, Nashville, TN 37235, USA

${ }^{3}$ Department of Physics, Fisk University, 1000 17th Avenue North, Nashville, TN 37208, USA

${ }^{4}$ Harvard-Smithsonian Center for Astrophysics, 60 Garden Street, Cambridge, MA 02138, USA

${ }^{5}$ Institute for Astronomy, University of Hawaii, 2680 Woodlawn Drive, Honolulu, HI 96822-1839, USA

${ }^{6}$ Department of Astronomy \& Astrophysics, The Pennsylvania State University, 525 Davey Lab, University Park, PA 16802, USA

${ }^{7}$ Center for Exoplanets and Habitable Worlds, The Pennsylvania State University, 525 Davey Lab, University Park, PA 16802, USA

${ }^{8}$ Department of Astronomy, University of California at Berkeley, Berkeley, CA 94720, USA

${ }^{9}$ NASA Ames Research Center, M/S 244-30, Moffett Field, CA 94035, USA

${ }^{10}$ Bay Area Environmental Research Institute, 625 2nd Street, Suite 209, Petaluma, CA 94952, USA

${ }_{11}^{11}$ NASA Goddard Space Flight Center, Greenbelt, MD 20771, USA

12 South African Astronomical Observatory, Cape Town, South Africa

${ }^{13}$ Las Cumbres Observatory Global Telescope Network, 6740 Cortona Drive, Suite 102, Santa Barbara, CA 93117, USA

${ }^{14}$ Department of Astronomy, The Ohio State University, Columbus, OH 43210, USA

${ }^{15}$ Perth Exoplanet Survey Telescope, Perth, Australia

${ }^{16}$ ICO, Adelaide, Australia

${ }^{17}$ American Association of Variable Star Observers, 49 Bay State Road, Cambridge, MA 02138, USA

${ }^{18}$ Hazelwood Observatory, Australia

${ }^{19}$ Department of Astronomy, California Institute of Technology, 1200 E. California Boulevard, MC 249-17, Pasadena, CA 91125, USA

${ }^{20}$ Jet Propulsion Laboratory, California Institute of Technology, 4800 Oak Grove Drive, Pasadena, CA 91109, USA

${ }^{21}$ Department of Astronomy, University of Washington, Box 351580, Seattle, WA 98195, USA

22 Observatoire Astronomique de l'Université de Genève, 51 ch. des Maillettes, 1290 Versoix, Switzerland

${ }^{23}$ Department of Astrophysical Sciences, Princeton University, Princeton, NJ 08544, USA

${ }^{24}$ Department of Physics and Astronomy, University of Louisville, Louisville, KY 40292, USA

${ }^{25}$ Department of Physics, Westminster College, New Wilmington, PA 16172, USA

${ }^{26}$ Department of Physics and Astronomy, Swarthmore College, Swarthmore, PA 19081, USA ${ }^{27}$ Ellinbank Observatory, Victoria, Australia

${ }^{28}$ Department of Physics and Astronomy, University of Pennsylvania, Philadelphia, PA 19104, USA

${ }^{29}$ School of Physics, University of New South Wales, Sydney, NSW 2052, Australia

${ }^{30}$ Australian Center for Astrobiology, University of New South Wales, Sydney, NSW 2052, Australia

${ }^{31}$ Computational Engineering and Science Research Centre, University of Southern Queensland, Toowoomba, Queensland 4350, Australia

${ }_{33}$ Department of Physics and Astronomy, University of Montana, Missoula, MT 59812, USA

${ }^{33}$ Department of Physics and Astronomy, Brigham Young University, Provo, UT 84602, USA

Received 2016 July 6; revised 2017 March 3; accepted 2017 March 6; published 2017 April 18

\begin{abstract}
We report the discovery of a transiting exoplanet, KELT-11b, orbiting the bright $(V=8.0)$ subgiant HD 93396. A global analysis of the system shows that the host star is an evolved subgiant star with $T_{\text {eff }}=5370 \pm 51 \mathrm{~K}$, $M_{*}=1.438_{-0.052}^{+0.061} M_{\odot}, R_{*}=2.72_{-0.17}^{+0.21} R_{\odot}, \log g_{*}=3.727_{-0.046}^{+0.040}$, and $[\mathrm{Fe} / \mathrm{H}]=0.180 \pm 0.075$. The planet is a lowmass gas giant in a $P=4.736529 \pm 0.00006$ day orbit, with $M_{P}=0.195 \pm 0.018 M_{\mathrm{J}}, R_{P}=1.37_{-0.12}^{+0.15} R_{\mathrm{J}}$, $\rho_{P}=0.093_{-0.024}^{+0.028} \mathrm{~g} \mathrm{~cm}^{-3}$, surface gravity $\log g_{P}=2.407_{-0.086}^{+0.080}$, and equilibrium temperature $T_{\mathrm{eq}}=1712_{-46}^{+51} \mathrm{~K}$. KELT-11 is the brightest known transiting exoplanet host in the southern hemisphere by more than a magnitude and is the sixth brightest transit host to date. The planet is one of the most inflated planets known, with an exceptionally large atmospheric scale height $(2763 \mathrm{~km})$, and an associated size of the expected atmospheric transmission signal of 5.6\%. These attributes make the KELT-11 system a valuable target for follow-up and atmospheric characterization, and it promises to become one of the benchmark systems for the study of inflated exoplanets.
\end{abstract}

Key words: planetary systems - planets and satellites: detection - stars: individual (KELT-11) techniques: photometric - techniques: radial velocities

Supporting material: data behind figure, machine-readable tables 


\section{Introduction}

The discovery of transiting exoplanets has been marked by two eras. The first era began with observations showing that the planet HD 209458b, first discovered by the radial velocity (RV) method, transited its host star (Henry et al. 2000; Charbonneau et al. 2000), and with the discovery of the first planet with the transit method, OGLE-TR-56b (Udalski et al. 2002; Konacki et al. 2003). This began a period of rapid discovery of new transiting exoplanets using small, automated, and dedicated telescopes, most notably by the HATNet (Bakos et al. 2004), SuperWASP (Pollacco et al. 2006), TrES (Alonso et al. 2004), and XO (McCullough et al. 2006) projects. The second era was marked by the 2007 launch of the CoRoT mission (Rouan et al. 1998), and then in 2009 with the launch of the Kepler mission (Borucki et al. 2010). Space-based detection of transiting planets was a huge leap forward, especially with the ability to detect smaller and longer-period transiting planets.

Although the Kepler mission has been tremendously fruitful with the number and variety of detected planets, especially for the determination of the underlying population and demographics of exoplanets, the small ground-based telescopes have continued to make many important discoveries. Notably, the population of transiting planets discovered by the ground-based surveys tend to be large planets with short orbital periods orbiting bright stars, due to selection and observation bias (Pepper et al. 2003; Gaudi 2005; Pepper \& Gaudi 2005; Pont et al. 2006; Fressin et al. 2007). These planets, unlike the vast majority of the Kepler planets, offer great potential for detailed characterization of the atmospheres of exoplanets. The bulk of our understanding of exoplanetary atmospheres comes from observations of planets with host stars with $V<13$ (Seager \& Deming 2010; Sing et al. 2016).

For that reason, the ongoing discoveries from ground-based transit surveys will continue to provide great value, at least until the launch of the Transiting Exoplanet Survey Satellite (TESS) mission (Ricker et al. 2015). Among these projects is the Kilodegree Extremely Little Telescope (KELT) survey. KELT fills a niche in planet discovery space by observing stars generally brighter than those observed by the other groundbased surveys, with a target magnitude regime of $7.5<V<10.5$. The KELT-North telescope (Pepper et al. 2007) has been operating since 2006, and has discovered 10 exoplanets to date.

The KELT-South telescope (Pepper et al. 2012) has been operating since 2009. It is located in Sutherland, South Africa, and surveys a large fraction of the southern hemisphere, where no transiting planets have been discovered with a host star brighter than $V=9.2$. KELT-South has discovered or codiscovered three transiting planets to date: KELT-10b (Kuhn et al. 2016), and WASP-122b/KELT-14b and KELT-15b (Rodriguez et al. 2016).

In this paper, we report the discovery of a new exoplanet, KELT-11b. The discovery of KELT-11b was enabled by a collaboration between the KELT team and the Retired A-star Program of the California Planet Search (CPS) team, an RV survey that has discovered 34 exoplanets (Johnson et al. 2011). This discovery attests to the value of combining data from multiple surveys to enable future discovery. This planet has one of the brightest host stars in the sky for a transiting planet, and is by far the brightest transit host in the southern hemisphere. It is also extraordinarily inflated and one of the lowest-density planets known.

\section{Discovery and Follow-up Observations}

In this section, we describe the KELT data and follow-up photometric and RV observations that led to the discovery and confirmation of KELT-11b. A detailed description of the KELT pipeline and candidate selection process can be found in Siverd et al. (2012) and Kuhn et al. (2016).

\subsection{KELT-South}

KELT- $11 \mathrm{~b}$ is located in the KELT-South field 23, which is centered at $\mathbf{J} 2000 \alpha=10^{\mathrm{h}} 43^{\mathrm{m}} 48^{\mathrm{s}}, \delta=-20^{\circ} 00^{\prime} 00^{\prime \prime}$. This field was monitored from UT 2010 March 12 to UT 2014 July 9, resulting in 3910 images after post-processing and removal of bad images. Following the same strategy as described in Kuhn et al. (2016), we reduced the raw images, extracted the light curves, and searched for transit candidates. One candidate from this processing, KS23C00790 (HD 93396, TYC 5499-1085-1, 2MASS J10464974-0923563), located at $\alpha=10^{\mathrm{h}} 46^{\mathrm{m}} 49^{\mathrm{s}} .7$, $\delta=-9^{\circ} 23^{\prime} 566^{\prime \prime} 5 \mathrm{~J} 2000$, was rated as a top candidate from the field. The host star properties can be seen in Table 1. We use the Box-fitting Least-squares algorithm (Kovács et al. 2002) as implemented in the VARTOOLS software package (Hartman \& Bakos 2016) to identify transit candidates, making use of statistics that come from the VARTOOLS package, as well as from Pont et al. (2006) and Burke et al. (2006). The associated selection criteria and the corresponding values for KELT-11b are shown in Table 2. For the choices of the particular selection criteria, refer to Siverd et al. (2012). The discovery light curve itself is shown in Figure 1, and the data are provided in Table 3.

\subsection{Photometric Follow-up}

We obtained follow-up time-series photometry of KELT-11b to check for false positives such as eclipsing binary stars, and to better determine the transit shape, depth, and duration. We used the TAPIR software package (Jensen 2013) to predict transit events, and we obtained nine full or partial transits in multiple bands between 2015 January and 2016 February. All data were calibrated and processed using the AstroImageJ package (AIJ $;{ }^{34}$ Collins \& Kielkopf 2013; Collins et al. 2017) unless otherwise stated. The follow-up light curves are displayed in Figure 2, and Table 4 lists the details of the observations. A binned combination of all of the follow-up light curves is shown in the bottom panel of Figure 2, though that combination is for display purposes and is not used for analysis.

It was extremely difficult to obtain reliable ground-based photometry, due to the combination of the shallow transit depth (about $2.5 \mathrm{mmag}$ ) and long duration $(7.3 \mathrm{hr}$ ). Any given observatory will have difficulty observing a full transit including out-of-transit baseline observations within a single night. In fact, KELT-11b is the longest-duration transiting planet discovered using the transit method from a ground-based facility, and the one with the shallowest transit as well. We managed to obtain one full transit, and a number of partial transits that covered ingresses and egresses. The discovery light curve can be accessed in the online journal in a machinereadable table linked to Table 3. All follow-up photometric observations are provided in the online journal in a combined machine-readable table linked to Figure 2.

\footnotetext{
${ }^{34}$ http://www.astro.louisville.edu/software/astroimagej
} 
Table 1

Stellar Properties of KELT-11

\begin{tabular}{|c|c|c|c|c|}
\hline $\begin{array}{l}\text { Parameter } \\
\text { Names }\end{array}$ & Description & $\begin{array}{c}\text { KELT-11 Value } \\
\text { HD 93396 } \\
\text { TYC 5499-1085-1 } \\
\text { 2MASS J10464974-0923563 }\end{array}$ & Source & Reference(s) \\
\hline$\alpha_{\mathrm{J} 2000}$ & Right ascension (R.A.) & $10: 46: 49.741$ & Tycho-2 & Høg et al. (2000) \\
\hline$\delta_{\mathrm{J} 2000}$ & Declination (decl.) & $-09: 23: 56.48$ & Tycho-2 & Høg et al. (2000) \\
\hline$B_{T}$ & Tycho $B_{T}$ magnitude & $9.072 \pm 0.018$ & Tycho-2 & Høg et al. (2000) \\
\hline$V_{T}$ & Tycho $V_{T}$ magnitude & $8.130 \pm 0.013$ & Tycho-2 & Høg et al. (2000) \\
\hline$V$ & Johnson $V$ magnitude & 8.03 & Hipparcos & Perryman et al (1997) \\
\hline$J$ & 2MASS magnitude & $6.616 \pm 0.024$ & 2MASS & Cutri et al. (2003), Skrutskie et al. (2006) \\
\hline$H$ & 2MASS magnitude & $6.251 \pm 0.042$ & 2MASS & Cutri et al. (2003), Skrutskie et al. (2006) \\
\hline$K_{S}$ & 2MASS magnitude & $6.122 \pm 0.018$ & 2MASS & Cutri et al. (2003), Skrutskie et al. (2006) \\
\hline$W 1$ & WISE passband & $6.152 \pm 0.1$ & WISE & Wright et al. (2010), Cutri et al. (2014) \\
\hline$W 2$ & WISE passband & $6.068 \pm 0.036$ & WISE & Wright et al. (2010), Cutri et al. (2014) \\
\hline W3 & WISE passband & $6.157 \pm 0.015$ & WISE & Wright et al. (2010), Cutri et al. (2014) \\
\hline W4 & WISE passband & $6.088 \pm 0.048$ & WISE & Wright et al. (2010), Cutri et al. (2014) \\
\hline$\mu_{\alpha}$ & Proper motion in R.A. $\left(\right.$ mas $\mathrm{yr}^{-1}$ ) & $-78.30 \pm 0.95$ & Tycho-2 & van Leeuwen (2007) \\
\hline$\mu_{\delta}$ & Proper motion in decl. $\left(\right.$ mas $\mathrm{yr}^{-1}$ ) & $-77.61 \pm 0.68$ & Tycho-2 & van Leeuwen (2007) \\
\hline$U^{\mathrm{a}}$ & Space motion $\left(\mathrm{km} \mathrm{s}^{-1}\right)$ & $-9.6 \pm 1.2$ & $\cdots$ & This work \\
\hline$V$ & Space motion $\left(\mathrm{km} \mathrm{s}^{-1}\right)$ & $-46.2 \pm 3.0$ & $\cdots$ & This work \\
\hline$W$ & Space motion $\left(\mathrm{km} \mathrm{s}^{-1}\right)$ & $-8.4 \pm 3.4$ & $\cdots$ & This work \\
\hline Distance & Distance $(\mathrm{pc})$ & $98 \pm 5$ & $\cdots$ & This work \\
\hline RV & Absolute RV $\left(\mathrm{km} \mathrm{s}^{-1}\right)$ & $35.0 \pm 0.1$ & $\ldots$ & This work \\
\hline$v \sin i_{*}$ & Stellar rotational velocity $\left(\mathrm{km} \mathrm{s}^{-1}\right)$ & $2.66 \pm 0.50$ & $\cdots$ & This work \\
\hline
\end{tabular}

Note.

${ }^{\text {a }} U$ is positive in the direction of the Galactic center.

Table 2

KELT-South Selection Criteria for Candidate KS23C00790

\begin{tabular}{llc}
\hline \hline Statistic & $\begin{array}{l}\text { Selection } \\
\text { Criteria }\end{array}$ & $\begin{array}{c}\text { Value for } \\
\text { KS23C00790/KELT-11 }\end{array}$ \\
\hline Signal detection efficiency & SDE $>7.0$ & 9.91663 \\
Signal to pink-noise & SPN $>7.0$ & 8.16926 \\
Transit depth & $\delta<0.05$ & 0.00344 \\
$\chi^{2}$ ratio & $\frac{\Delta \chi^{2}}{\Delta \chi_{-}^{2}}>1.5$ & 1.808 \\
Duty cycle & $q<0.1$ & 0.05333 \\
\hline
\end{tabular}

\subsubsection{Westminster College Observatory (WCO)}

We observed an ingress of KELT-11b from the WCO, PA, on UT 2015 January 1 in the $I$ filter. The observations employed a $0.35 \mathrm{~m} f / 11$ Celestron C14 Schmidt-Cassegrain telescope and SBIG STL-6303E CCD with a $3 \mathrm{k} \times 2 \mathrm{k}$ array of $9 \mu \mathrm{m}$ pixels, yielding a $24^{\prime} \times 16^{\prime}$ field of view and $11^{\prime \prime} 4$ pixel $^{-1}$ image scale at $3 \times 3$ pixel binning.

\subsection{2. $M O R C$}

We observed a partial transit of KELT- $11 \mathrm{~b}$ using an $0.6 \mathrm{~m}$ RCOS telescope at the Moore Observatory (MORC), operated by the University of Louisville. The telescope has an Apogee $\mathrm{U} 16 \mathrm{M} 4 \mathrm{~K} \times 4 \mathrm{~K} C \mathrm{CD}$, giving a $26^{\prime} \times 26^{\prime}$ field of view and 0 ". 39 pixel $^{-1}$. We observed the transit on UT 2015 February 08 in alternating Sloan $g$ and $i$ filters from before the ingress and past the mid-transit.

\subsubsection{MINERVA}

We observed a transit of KELT-11b in the Sloan $i$-band using one of the MINERVA Project telescopes (Swift et al.
2015) on the night of UT 2015 February 08. MINERVA used four $0.7 \mathrm{~m}$ PlaneWave CDK-700 telescopes that are located on Mt. Hopkins, Arizona, at the Fred L. Whipple Observatory. While the four telescopes are normally used to feed a single spectrograph to discover and characterize exoplanets through RV measurements, for the KELT-11 observations, we used a single MINERVA telescope in its photometric imaging mode. That telescope had an Andor iKON-L $2048 \times 2048$ camera, which gave a field of view of $20 ! 9 \times 20^{\prime} \cdot 9$ and a plate scale of $0{ }^{\prime \prime} 6 \mathrm{pixel}^{-1}$. The camera has a $2048 \times 2048$ back-illuminated deep depletion sensor with fringe suppression. Due to the brightness of KELT-11, we heavily defocused for our observations, such that the image of KELT-11 was a "donut" approximately 20 pixels in diameter.

\subsubsection{Perth Exoplanet Survey Telescope (PEST) Observatory}

On UT 2015 March 08, we observed a partial transit from the PEST Observatory, located in Perth, Australia. The observations were taken with a $0.3 \mathrm{~m}$ Meade LX200 telescope working at $f / 5$, and with a $31^{\prime} \times 21^{\prime}$ field of view. The camera is an SBIG ST-8XME, with $1530 \times 1020$ pixels, yielding 1!"2 pixel $^{-1}$. An ingress was observed using a Cousins $I$ filter.

\subsubsection{Ivan Curtis Observatory (ICO)}

On UT 2015 March 03, we observed a partial transit at the ICO, located in Adelaide, Australia. The observations were taken with a $0.235 \mathrm{~m}$ Celestron Schmidt-Cassegrain telescope with an Antares 0.63x focal reducer, giving an overall focal ratio of $f / 6.3$. The camera is an Atik 320e, which uses a cooled Sony ICX274 CCD of $1620 \times 1220$ pixels. The field of view is $16 ! 6 \times 12 ! 3$, with a resolution of 0 !' 62 pixel $^{-1}$. An egress was observed using a Johnson $R$ filter. 


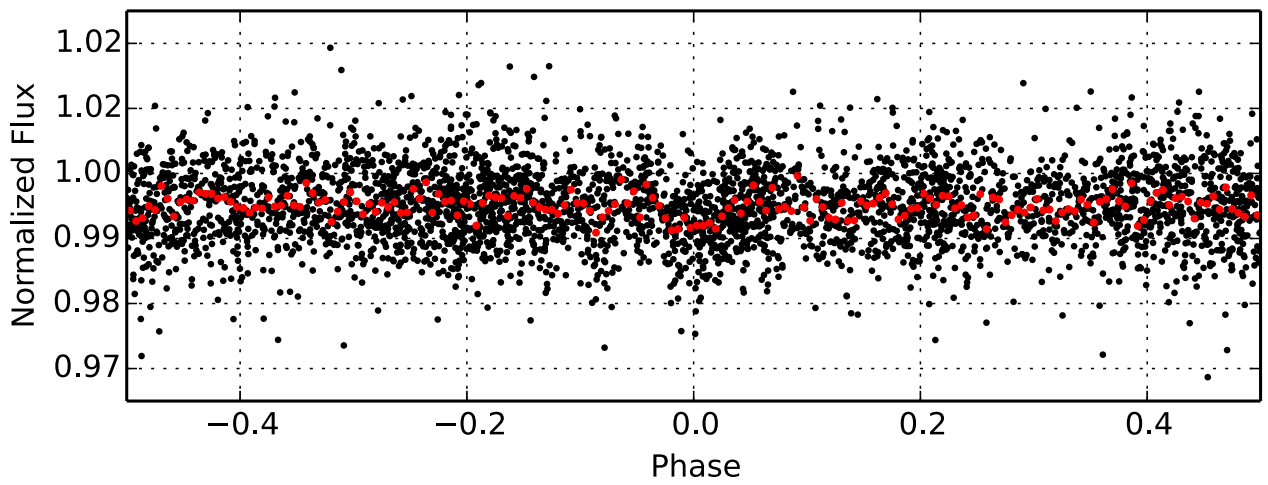

Figure 1. Discovery light curve of KELT-11b from the KELT-South telescope. The light curve contains 3910 observations spanning just over four years, phase folded to the orbital period of $P=4.7360001$ days; the red points show the light curve binned in phase using a bin size of 0.005 in phase.

Table 3

Discovery Light Curve from KELT

\begin{tabular}{lcc}
\hline \hline Time $\left(\mathrm{JD}_{T T}\right)$ & Relative Mag & Relative Mag Error \\
\hline 2455268.287210 & -0.0189 & 0.0022 \\
2455268.316559 & -0.0052 & 0.0022 \\
2455268.334478 & -0.0104 & 0.0022 \\
2455268.352391 & -0.0044 & 0.0023 \\
2455268.363761 & 0.0024 & 0.0021 \\
2455268.379669 & 0.0046 & 0.0021 \\
2455268.397738 & -0.0009 & 0.0023 \\
2455268.416282 & -0.0029 & 0.0019 \\
2455268.441287 & 0.0008 & 0.0019 \\
2455268.457047 & 0.0037 & 0.0019 \\
\hline
\end{tabular}

(This table is available in its entirety in machine-readable form.)

\subsubsection{Peter van de Kamp Observatory}

We observed an ingress in the Sloan $z$-band at the Swarthmore College Peter van de Kamp Observatory (PvdK) on 2015 March 18. The observatory uses a $0.6 \mathrm{~m}$ RCOS Telescope with an Apogee U16M $4 \mathrm{~K} \times 4 \mathrm{~K} \mathrm{CCD,} \mathrm{giving} \mathrm{a}$ $26^{\prime} \times 26^{\prime}$ field of view. Using $2 \times 2$ binning, it has 0 "'76 pixel $^{-1}$.

\subsubsection{Las Cumbres Observatory Global Telescope (LCOGT)-CPT}

We observed an egress of KELT-11b in the Sloan $i$-band during bright time on UT 2015 May 04, using one of the $1 \mathrm{~m}$ telescopes in the LCOGT network ${ }^{35}$ located at the South African Astronomical Observatory (SAAO) in Sutherland, South Africa. The LCOGT telescopes at SAAO have $4 \mathrm{~K} \times 4 \mathrm{~K}$ SBIG Science cameras and offer a $16^{\prime} \times 16^{\prime}$ field of view and an unbinned pixel scale of 0 !" $23 \mathrm{pixel}^{-1}$.

\subsubsection{Manner-Vanderbilt Ritchey-Chrétien (MVRC)}

We observed one full transit of KELT-11b using the MVRC telescope located at the Mt. Lemmon summit of the Steward Observatory, Arizona, on UT 2016 February 22 in the $r^{\prime}$ filter. The observations employed a $0.6 \mathrm{~m} \mathrm{f} / 8 \mathrm{RC}$ Optical Systems Ritchey-Chrétien telescope and SBIG STX-16803 CCD with a $4 \mathrm{k} \times 4 \mathrm{k}$ array of $9 \mu \mathrm{m}$ pixels, yielding a $26 ! 6 \times 26$ ! 6 field of view and 0. " 39 pixel $^{-1}$ image scale. The telescope was heavily

\footnotetext{
35 http://lcogt.net/
}

defocused, resulting in a typical "donut" shaped stellar PSF with a diameter of $\sim 25^{\prime \prime}$.

\subsection{Spectroscopic Follow-up}

We obtained spectroscopic observations of KELT-11 to measure the RV orbit of the planet, and to measure the parameters of the host star. The observations that were used to derive stellar parameters are listed in Table 5. The observations that provide RV measurements are listed in Table 6 and are displayed in Figure 3.

\subsubsection{Tillinghast Reflector Echelle Spectrograph (TRES)}

In order to measure the host star properties, we obtained a spectrum with TRES, on the $1.5 \mathrm{~m}$ telescope at the Fred Lawrence Whipple Observatory (FLWO) on Mt. Hopkins, Arizona, on UT 2015 January 28. The spectrum has a resolution of $R=44,000$, a signal-to-noise ratio $(\mathrm{S} / \mathrm{N})=100.4$, and was extracted as described in Buchhave et al. (2010).

\subsubsection{KECK High Resolution Echelle Spectrometer (HIRES)}

Well before KELT observations of this star began, the RV of HD 93396 had been monitored at the Keck Observatory using HIRES (Vogt et al. 1994) starting in 2007 as part of the "Retired A Stars" program (Johnson et al. 2006, 2011). Observations were conducted using the standard setup of the California Planet Survey (Howard et al. 2010; Johnson et al. 2010) using the B5 decker and the iodine cell. RV measurements were made with respect to a high $\mathrm{S} / \mathrm{N}$, iodine-free template observation (Butler et al. 1996), which we also use to measure the stellar properties. Exposure times ranged from 50 to $120 \mathrm{~s}$ depending on the seeing, with an exposure meter ensuring that all exposures reached $\mathrm{S} / \mathrm{N} \approx 150$ per pixel at $550 \mathrm{~nm}$.

\subsubsection{Automated Planet Finder (APF)}

To supplement the HIRES RV spectra, we also observed KELT-11 with the Levy spectrograph on the APF telescope at Lick Observatory. We collected 16 RV measurements between 2015 January 12 and 2015 November 4. The observational setup was similar to the setup used for the APF observations described in Fulton et al. (2015a). We observed the star through a cell of gaseous iodine using the standard $1^{\prime \prime} \times 3^{\prime \prime}$ slit for a spectral resolution of $R \approx 100,000$, and collected an iodinefree template spectrum using the 0 ." $75 \times 8^{\prime \prime}$ slit $(R \approx 120,000$, Vogt et al. 2014). As with the Keck/HIRES velocities, the RVs 


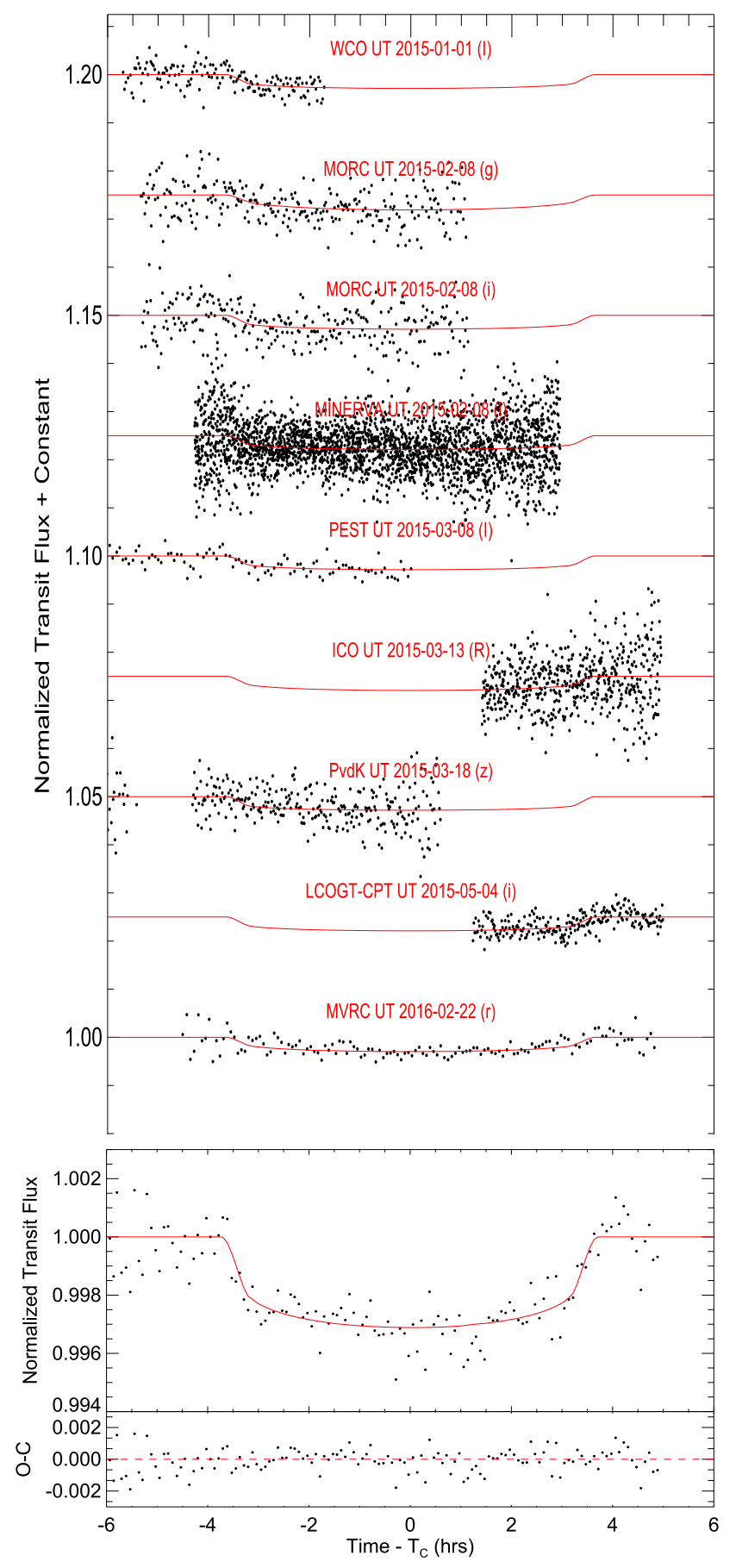

Figure 2. (Top) The individual follow-up observations of KELT-11b from the KELT Follow-up Network. The global model with limb-darkening coefficients corresponding to the filter band is over-plotted on each light curve in red. (Bottom) All follow-up transit combined and binned in 5 minute intervals to best represent the transit features. This plot is not used in the analysis and is only for display. The combined and binned models from each transit are represented by the red line. The light curve photometry is available in machinereadable form in the online journal. The data used to create this figure are available.

are measured using a forward modeling process that fits for the Doppler shift of the deconvolved stellar template with respect to the stationary forest of molecular iodine absorption lines, while simultaneously modeling the instrumental point-spread function (Butler et al. 1996). The photon-weighted mid-exposure times are calculated using a software-based exposure meter that measures the real-time photon flux received on the guider camera during each spectral exposure (Kibrick et al. 2006). Exposure times ranged from 18 to 30 minutes depending on seeing and transparency to obtain $\mathrm{S} / \mathrm{N} \approx 100$ pixel $^{-1}$ at $550 \mathrm{~nm}$.

\subsubsection{Bisector Spans (BSs)}

We calculate BSs for each APF spectra following the prescription of Fulton et al. (2015a). We cross-correlate each spectrum with a synthetic stellar template interpolated from the Coelho (2014) grid of stellar atmosphere models to match the adopted stellar parameters. We restrict the BS analysis to the 15 echelle orders spanning 4260-5000 $\AA$ in order to avoid iodine contamination and telluric lines, and to reduce the effect of telescope guiding errors on the instrumental PSF. The blue orders were used where the seeing is generally worse and the slit is more uniformly illuminated. This reduces false bisector variations caused by changes in the slit illumination. The BS is a measure of the line asymmetry calculated as the difference in the midpoint of line segments drawn between the 65th and 95th percentile levels of the CCF. The reported BSs and uncertainties are the mean and standard deviation on the mean over the 15 spectral orders analyzed. The BS values are included in Table 6, and are plotted against the RV values in Figure 4.

\subsection{High-contrast Imaging}

We observed KELT-11 with Adaptive Optics (AO) imaging to identify any faint, nearby companions to the host star. The observations were performed at Palomar Observatory's Hale telescope on 2015 February 7 using the Stellar Double Coronagraph (Bottom et al. 2015) operating with a ringapodized vortex. Observations were taken in the $K$-short band, obtaining 60 exposures at $29.7 \mathrm{~s}$ each. Figure 5 shows the image of the star, and Figure 6 shows the associated contrast curve. No astrophysical sources are seen near KELT-11. At separations of 1.5-4 arcsec, which at the distance of KELT-11 corresponds to a projected separation of about 14-98 au, we can exclude companions with flux ratios of $4 \times 10^{-4}$ compared to KELT-11, which includes stars down to mid-Mtype dwarfs.

\section{Analysis and Results}

\subsection{Stellar Parameters from Spectra}

To obtain a thorough picture of the stellar parameters, we used multiple parameter extraction techniques with different spectra to obtain consistent and robust results.

We applied the spectral parameter classification (SPC; Buchhave et al. 2012) technique to the spectrum from TRES, with $T_{\text {eff }}, \log g_{*},[m / H]$, and $v \sin I_{*}$ as free parameters. SPC cross-correlates an observed spectrum against a grid of synthetic spectra based on Kurucz atmospheric models (Kurucz 1992). The resulting parameters are $T_{\text {eff }}=5390 \pm 50 \mathrm{~K}, \log g_{*}=$ $3.789 \pm 0.100,[m / H]=0.189 \pm 0.080$, and $v \sin I_{*}=2.66 \pm$ $0.50 \mathrm{~km} \mathrm{~s}^{-1}$. These parameters match the identification of KELT-11 from Houk \& Swift (1999) as a G8/K0 IV spectral type.

We then independently analyzed the Keck and APF template spectra using a modified version of the SpecMatch pipeline (Petigura et al. 2015) as described in Fulton et al. (2015b). 
Table 4

Record of the Photometric Follow-up Observations and Detrending Parameters Used by AIJ for the Global Fit

\begin{tabular}{|c|c|c|c|c|c|c|}
\hline Observatory & Date (UT) & Filter & FOV & Pixel Scale & Exposure (s) & Detrending Parameters for Global Fit \\
\hline WCO & UT 2015 Jan 01 & $I$ & $24^{\prime} \times 16^{\prime}$ & $1 ! 4$ & binned $4 \times 10$ & Airmass, Time \\
\hline MORC & UT 2015 Feb 08 & $g^{\prime}$ & $26^{\prime} 6 \times 26^{\prime} 6$ & 0 " 39 & 20 & Airmass, Time \\
\hline MORC & UT 2015 Feb 08 & $i^{\prime}$ & $26^{\prime} 6 \times 26^{\prime} 6$ & 0 " 39 & 20 & Airmass, Time \\
\hline MINERVA & UT 2015 Feb 08 & $i^{\prime}$ & $20^{\prime} 9 \times 20^{\prime} .9$ & $0 " .6$ & 6 & Airmass, Time \\
\hline PEST & UT 2015 Mar 08 & $I$ & $31^{\prime} \times 21^{\prime}$ & $1 ! 2$ & binned $8 \times 15$ & Airmass, Time \\
\hline $\mathrm{ICO}$ & UT 2015 Mar 13 & $R$ & $16 ! 6 \times 12 ! 3$ & $0 " .62$ & 20 & Airmass, Time \\
\hline PvdK & UT 2015 Mar 18 & $z^{\prime}$ & $26^{\prime} \times 26^{\prime}$ & $0 ! 76$ & 45 & Airmass, Time \\
\hline LCOGT (CPT) & UT 2015 May 04 & $i^{\prime}$ & $15 ! 8 \times 15^{\prime} .8$ & 0.23 & 12 & Airmass, Time \\
\hline MVRC & UT 2016 Feb 22 & $r^{\prime}$ & $26^{\prime} 6 \times 26^{\prime} 6$ & 0 " 39 & binned $6 \times 30$ & Airmass \\
\hline
\end{tabular}

SpecMatch fits a grid of model stellar atmospheres (Coelho 2014) to large regions of the observed spectrum to measure $T_{\text {eff }}$, $\log g_{*}$, and $[\mathrm{Fe} / \mathrm{H}]$. Our only modification to the code is to employ the ExoPy differential-evolution Markov Chain Monte Carlo (MCMC) fitting engine (Fulton et al. 2013) in place of $\chi^{2}$ minimization to find the optimal solution. We calibrate the $T_{\text {eff }}$ and $\log g_{*}$ values produced by SpecMatch to show good agreement with the values of Valenti \& Fischer (2005) for the 353 stars in their sample that were observed on Keck. Values of $\log g_{*}$ are calibrated against the stars from the Huber et al. (2012) sample with asteroseismically measured $\log g_{*}$. This calibration is done separately for both APF and Keck spectra using our modified version of the pipeline. However, the faint Kepler stars from the (Huber et al. 2012) sample have not been observed on APF so the $\log g_{*}$ calibration is bootstrapped from stars that have been observed at both Keck and APF. Parameter uncertainties are determined by measuring the scatter of our calibrated parameters against the values form Valenti \& Fischer (2005) and Huber et al. (2012).

The best-fit spectroscopic parameters measured from all three reductions are listed in Table 7.

\subsection{Spectral Energy Distribution (SED) Analysis}

We estimate the distance and reddening to KELT-11 by fitting Kurucz (1979) stellar atmosphere models to the SED from catalog broadband photometry. The available catalog photometry spans the range from the GALEX FUV band at $0.16 \mu \mathrm{m}$ to the WISE $22.8 \mu \mathrm{m}$ band (see Table 1).

We fix $T_{\text {eff }}, \log g_{*}$, and $[\mathrm{Fe} / \mathrm{H}]$ to the TRES-derived values from Section 3.1, and leave the distance $(d)$ and reddening $\left(A_{V}\right)$ as free parameters. We restricted $A_{V}$ to be at most $0.11 \mathrm{mag}$, as determined from the maximum line-of-sight extinction from the dust maps of Schlegel et al. (1998). The catalog photometric and associated model fit are displayed in Figure 7. We find best-fit parameters of $A_{V}=0.09_{-0.05}^{+0.02} \mathrm{mag}$ and $d=98 \pm 5 \mathrm{pc}$. This agrees quite well with, and is consistent with, the distance of $102 \pm 9 \mathrm{pc}$ from the Hipparcos parallax. The reduced $\chi^{2}$ of the fit is 7.0 when including the GALEX FUV and NUV photometry, but 1.9 when excluding the GALEX photometry. This reflects the apparent excess in the GALEX bands, suggestive of chromospheric activity. While it may be surprising to see such activity, similarly chromospherically active subgiants are rare but are seen in previous surveys of activity among subgiants (Jenkins et al. 2011). We looked for evidence of chromospheric activity in the $\mathrm{Ca} \mathrm{H}$ and $\mathrm{K}$ lines of the APF spectra and did not find any. Since the UV excess apparent in the SED is not corroborated by the $\mathrm{Ca}$ II $\mathrm{H}$ and $\mathrm{K}$ spectroscopy, we do not use the UV flux excess for any other aspects of our analysis or interpretations.
One possible cause of excess chromospheric activity can be spin-up of the host star from a dynamically interacting planet. We can explore that possibility in this case since we have a spectroscopic $v \sin I_{*}$ measurement. Assuming the star and planet are not highly misaligned, and therefore that $\sin I_{*} \sim 1$, we have a rotational period for the star of roughly 52 days. This is much longer than the orbital period of the planet, and so we do not find evidence for an unusually high rotational velocity due to planet spin-up of the star.

Using the Hipparcos distance and the spectroscopic $T_{\text {eff }}$, we can measure the stellar radius independently by summing the SED flux and solving the Stefan-Boltzmann relation. The summed bolometric flux received at Earth is $1.85 \pm 0.06 \times 10^{-8} \mathrm{erg} \mathrm{s}^{-1} \mathrm{~cm}^{-2}$, and the resulting stellar radius is $2.84 \pm 0.28 R_{\odot}$, which we use as a prior on the global analysis of the transit (see Section 3.5).

\subsection{Evolutionary Analysis}

We estimate the age of KELT-11 by fitting Yonsei-Yale isochrones to the values of $T_{\text {eff }}, \log g_{*}$, and $[\mathrm{Fe} / \mathrm{H}]$ given in Table 8 . We fix the stellar mass to the value of $1.438 M_{\odot}$ as listed in Table 8 (see Section 3.8 for a detailed discussion of the determination of that mass value). Our best-fit stellar parameters indicate that the star happens to fall on a rapid part of the evolutionary track, namely, the so-called "Hertzsprung gap" prior to the star's ascent up the red giant branch (see Figure 8). This permits a relatively precise, though modeldependent, age estimate, and we quote an age range of $3.52-3.54$ Gyr that spans the uncertainty in $T_{\text {eff }}$ and $\log g_{*}$.

\subsection{UVW Space Motion}

We examined the three-dimensional space motion of KELT11 to determine whether its kinematics match that of one of the main stellar populations of the Galaxy. The proper motion is

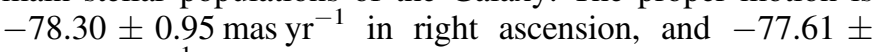
0.68 mas yr $^{-1}$ in declination (van Leeuwen 2007). The values for the absolute heliocentric RV as measured by the spectroscopic observations from Keck and APF (see Sections 2.3.2 and 2.3.3) are mutually consistent, and we adopt the value from the Keck results, which is $35.0 \pm 0.1 \mathrm{~km} \mathrm{~s}^{-1}$. The distance to the system as derived in Section 3.2 is $98 \pm 5 \mathrm{pc}$. These values transform to $U, V, W$ space motions with respect to the local standard of rest (LSR) of $-9.6 \pm 1.2,-46.2 \pm 3.0$, and $-8.4 \pm 3.4 \mathrm{~km} \mathrm{~s}^{-1}$, respectively. When calculating the UVW motion, we have accounted for the peculiar velocity of the Sun with respect to the LSR, using $U=8.5 \mathrm{~km} \mathrm{~s}^{-1}$, $V=13.38 \mathrm{~km} \mathrm{~s}^{-1}$, and $W=6.49 \mathrm{~km} \mathrm{~s}^{-1}$ from Coşkunoğlu et al. (2011). These values are consistent with that of a fastmoving thin disk star (Bensby et al. 2003). 
Table 5

Spectroscopic Follow-up Observations

\begin{tabular}{llccc}
\hline \hline Telescope/Instrument & Date Range & Resolution & Wavelength Range & Mean S/N \\
\hline FLWO 1.5 m/TRES & UT 2015 Jan 28 & $\approx 44,000$ & $3900-8900 \AA$ & Epochs \\
KECK/HIRES & UT 2007 Apr 26-UT 2015 Feb 08 & $\approx 55,000$ & $3640-7990 \AA$ & $\sim 100$ \\
APF/Levy & UT 2015 Jan 12-UT 2015 Nov 4 & $\approx 100,000$ & $3740-9700 \AA$ & $\sim 150$ \\
\\
\hline
\end{tabular}

\subsection{EXOFAST Global Fit}

Using a modified version of the IDL exoplanet fitting package, EXOFAST (Eastman et al. 2013), we perform a simulataneous MCMC analysis on the KECK HIRES and APF $\mathrm{RV}$ measurements, and the follow-up photometric observations by the KELT Follow-up Network. See Siverd et al. (2012) for a more complete description of the global modeling. For KELT$11 \mathrm{~b}$, we use either the Yonsei-Yale stellar evolution models (Demarque et al. 2004) or the Torres relations (Torres et al. 2010) to constrain $M_{\star}$ and $R_{\star}$. Our SED analysis (Section 3.2) of the catalog photometry of KELT-11 yielded an observed bolometric flux from the star of $1.82 \times 10^{-8} \mathrm{erg} \mathrm{s}^{-1} \mathrm{~cm}^{-2}$. We use the resulting stellar radius of $R_{*}=2.8 \pm 0.3 R_{\odot}$ as a prior in the global fit. From the KELT discovery data and follow-up photometric and spectroscopic observations, we set a prior on the host star's effective temperature $\left(T_{\text {eff }}=5391 \pm 50 \mathrm{~K}\right)$ and metallicity $([\mathrm{Fe} / \mathrm{H}]=0.189 \pm 0.08)$. All priors have Gaussian distributions.

The raw light curve and detrending parameters (see Table 4 for detrending parameters of each data set) are inputs into the final global fit using EXOFAST. For KELT-11b, we ran four separate EXOFAST fits using either the Torres relations or Yonsei-Yale stellar evolution models. For each stellar constraint, we ran one fit where the eccentricity of the planet's orbit was a free parameter and another where it was fixed at an eccentricity of zero. The results of these fits are shown in Tables 8 and 9. In Table 9, the quantities $T_{C, 1}, T_{C, 2}$, etc., refer to the determination of the transit center time for each of the follow-up light curves used in the model, shown in Figure 2. Because all but one of our follow-up photometry light curves are partial transits, we do not fit for transit timing variations as part of the global fit. The secondary eclipse parameters in all cases are not directly measured, but are rather based on the global fit. All four global fits are consistent with each other to within $2 \sigma$. For our overall analysis and discussion, we adopt the Yonsei-Yale circular fit parameters. We select Yonsei-Yale mainly because there are no stars in the sample from which the Torres relations were derived with the combination of $T_{\text {eff }}$ and $\log g_{*}$ that KELT-11 has, and with the short orbital period, there is a strong prior of zero eccentricity. However, since all fits yield similar results, we do not believe the particular choice significantly affects the overall system parameters.

Our stellar parameter priors strongly favor young, pre-main sequence stars, and also imply a mass prior strongly peaked at $1 M_{\odot}$ with a long tail toward higher masses. However, our global fit, due to the stellar density constraint from the transit, excludes the peak of this prior with high confidence, enhancing the importance of the high mass tail. The prior around the value of the ultimately inferred mass is biased slightly toward lowermass stars relative to a flat, "uninformative" prior. We believe this is justified because generally lower-mass stars with temperatures and surface gravities consistent with those measured for KELT-11 are favored both because higher-mass stars are rarer and because they spend less time in this region of the HR diagram.

We find a negative slope in the RV observations of $-0.006 \pm 0.0015 \mathrm{~m} \mathrm{~s}^{-1}$ day $^{-1}$. That is most likely a result of an outer massive companion in orbit around KELT-11. Since there is no evidence of significant curvature in the slope, the companion must have an orbital period longer than about 20 years. The companion could be a large planet or a brown dwarf, but it likely cannot be stellar mass, since there are no signs of an additional object in the spectral analysis. As noted in Section 2.4, there are no indications of a companion in the AO observations, though those observations are most sensitive at wide separations from the star.

\subsection{Irradiation History}

We find that KELT-11b is a highly inflated planet, joining the ranks of other gas giant planets that manifest radii much larger than predicted by standard models of irradiated objects with Jovian masses, which do not invoke additional sources of energy deposition in the interior of the planet. Several authors (e.g., Demory \& Seager 2011) have suggested an empirical insolation threshold $\left(\approx 2 \times 10^{8} \mathrm{erg} \mathrm{s}^{-1} \mathrm{~cm}^{-2}\right)$ above which gas giants exhibit increasing amounts of radius inflation. KELT$11 \mathrm{~b}$ clearly lies above this threshold, with a current estimated insolation of $1.95_{-0.20}^{+0.24} \times 10^{9} \mathrm{erg} \mathrm{s}^{-1} \mathrm{~cm}^{-2}$ and therefore its currently large inflated radius is not surprising. At the same time, the KELT-11 host star is found to currently be in a very rapid state of evolution, such that its radius is rapidly expanding as the star crosses the Hertzsprung gap toward the red giant branch. This means that the star's surface is rapidly encroaching on the planet, which presumably is rapidly driving up the planet's insolation and also the rate of any tidal interactions between the planet and the star.

Therefore it is interesting to consider whether KELT-11b's incident radiation from its host star has been below the empirical radius inflation threshold in the past. If KELT-11b's insolation only recently exceeded the inflation threshold, the system could then serve as an empirical testbed for the different timescales predicted by different inflation mechanisms (see, e.g., Assef et al. 2009; Spiegel \& Madhusudhan 2012).

To investigate this, we follow Penev et al. (2014) to simulate the past and future evolution of the star-planet system, using the measured parameters listed in Tables 8 and 9 as the presentday boundary conditions. This analysis is not intended to examine any type of planet-planet or planet-disk migration effects. Rather, it is a way to investigate the change in insolation of the planet over time due to the changing luminosity of the star and changing star-planet separation. We include the evolution of the star, assumed to follow the Yonsei-Yale stellar model with mass and metallicity as in Table 8. For simplicity, we assume that the stellar rotation is negligible and treat the star as a solid body. We also assume a circular orbit aligned with the stellar equator throughout the full analysis. The results of our simulations are shown in Figure 9. 

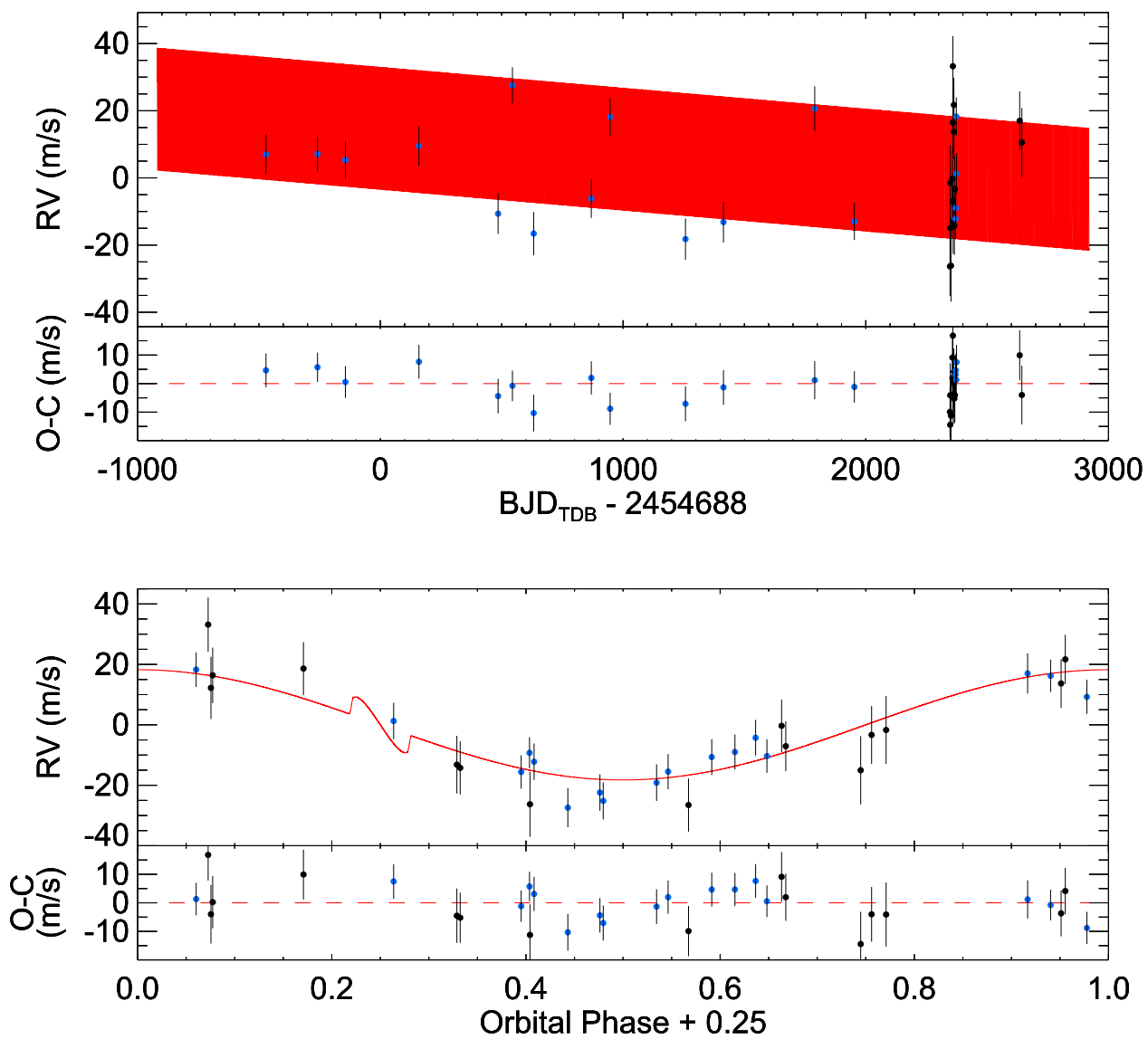

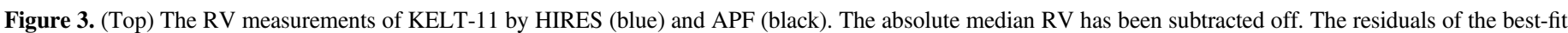

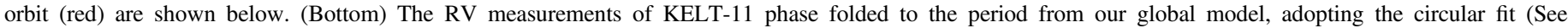

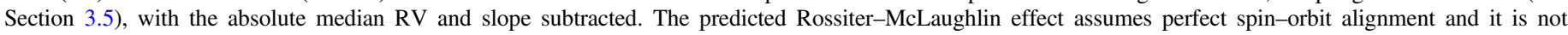
constrained by our data.

We tested a range of values for the tidal quality factor of the star $Q_{\star}^{\prime}$, from $\log Q_{\star}^{\prime}=6$ to $\log Q_{\star}^{\prime}=8$ (assuming a constant phase lag between the tidal bulge and the star-planet direction). $Q_{\star}^{\prime}$ is defined as the tidal quality factor divided by the Love number (i.e., $Q_{\star}^{\prime}=Q_{\star} / k_{2}$ ).

We find that in all cases the planet has always received more than enough flux from its host to keep the planet irradiated beyond the insolation threshold identified by Demory \& Seager (2011).

\subsection{False Positive Analysis}

We examine several lines of evidence to explore whether the combination of observations of this system are caused by a false positive, such as a blended eclipsing binary. First, we verify that all photometric observations of the transit are consistent with achromatic transit depths (see Section 2.2), since wavelength-dependent transit depths at the level detectable by our photometry would indicate that this is a blended eclipsing binary. We then check that in the KELT-11 spectra there are no signs of a second stellar spectrum blended with the spectrum of the host star (see Section 2.3), and we also see no faint companions blended with KELT-11b in the AO observations (Section 2.4). We also find no correlation between the BSs and the measured RVs (see Figure 4). The stellar surface gravity $\left(\log (g)=3.727_{-0.046}^{+0.040}\right)$ derived from the transits in the global fit, with no spectroscopic $\log g_{*}$ prior imposed, is consistent with the three spectroscopically derived $\log g_{*}$ values (see Table 7 ) within $\approx 1 \sigma$. We therefore conclude that KELT-11b is a bona fide planet.

\subsection{The Mass of KELT-11}

We find that the KELT-11 host star is located in a relatively sparsely populated part of the Hertzsprung-Russell (H-R) diagram, namely the "Hertzsprung gap." This is the part of the $\mathrm{H}-\mathrm{R}$ diagram, where relatively massive stars $\left(M_{*} \gtrsim 1.5 M_{\odot}\right)$ have finished core hydrogen fusion, but have yet to initiate hydrogen shell fusion, i.e., they have yet to reach the giant branch. In other words, this region is typically populated by relatively massive subgiants. This part of the diagram is sparsely populated because massive stars spend a very small amount of time in this phase of their evolution relative to the duration of their hydrogen main sequence or giant branch evolutionary states, and because massive stars are intrinsically more rare than less massive stars. It is precisely the short duration of this massive star's subgiant phase that allows us to put such a precise (albeit model-dependent) constraint on the age of the star.

However, the rapid evolution through this part of the $\mathrm{H}-\mathrm{R}$ diagram and the relative paucity of massive stars also implies that it is a priori unlikely that we would have found a transiting planet orbiting such a star. We therefore must be more diligent than usual in justifying our claims of the estimated parameters 
Table 6

KELT-11 RV Observations with HIRES and APF

\begin{tabular}{|c|c|c|c|c|c|}
\hline $\mathrm{BJD}_{\mathrm{TDB}}$ & $\begin{array}{c}\mathrm{RV} \\
\left(\mathrm{m} \mathrm{s}^{-1}\right)\end{array}$ & $\begin{array}{l}\text { RV Error } \\
\left(\mathrm{m} \mathrm{s}^{-1}\right)\end{array}$ & $\begin{array}{l}\begin{array}{l}\text { Bisector } \\
\left(\mathrm{m} \mathrm{s}^{-1}\right)\end{array}\end{array}$ & $\begin{array}{c}\text { Bisector Error } \\
\left(\mathrm{m} \mathrm{s}^{-1}\right)\end{array}$ & $\overline{\text { Instrument }}$ \\
\hline 2454216.875149 & 4.4 & 1.5 & $\ldots$ & $\ldots$ & HIRES \\
\hline 2454429.129861 & 4.5 & 1.3 & $\ldots$ & $\ldots$ & HIRES \\
\hline 2454847.046979 & 6.9 & 1.5 & $\ldots$ & $\ldots$ & HIRES \\
\hline 2455173.107559 & -13.3 & 1.5 & $\ldots$ & $\ldots$ & HIRES \\
\hline 2455232.144465 & 25.0 & 1.4 & $\cdots$ & $\cdots$ & HIRES \\
\hline 2455634.925941 & 15.5 & 1.4 & $\ldots$ & $\ldots$ & HIRES \\
\hline 2455945.177177 & -20.9 & 1.6 & $\ldots$ & $\ldots$ & HIRES \\
\hline 2456101.742516 & -15.8 & 1.5 & $\ldots$ & $\ldots$ & HIRES \\
\hline 2456477.738143 & 18.0 & 1.7 & $\ldots$ & $\ldots$ & HIRES \\
\hline 2456641.045559 & -15.6 & 1.4 & $\ldots$ & $\ldots$ & HIRES \\
\hline 2457057.922673 & -14.8 & 1.5 & $\ldots$ & $\ldots$ & HIRES \\
\hline 2457035.833572 & -12.5 & 3.7 & -4.8 & 6.7 & APF \\
\hline 2457035.957552 & 0.8 & 3.7 & -9.6 & 6.8 & APF \\
\hline 2457038.956627 & -23.8 & 3.5 & -4.8 & 4.8 & APF \\
\hline 2457044.920751 & 2.2 & 2.9 & 2.5 & 2.2 & APF \\
\hline 2457044.941030 & -4.6 & 2.7 & 3.1 & 4.7 & APF \\
\hline 2457046.859494 & 35.6 & 2.9 & 13.4 & 8.8 & APF \\
\hline 2457046.881613 & 18.9 & 3.0 & -1.3 & 7.3 & APF \\
\hline 2457048.072907 & -10.7 & 3.1 & -5.8 & 5.2 & APF \\
\hline 2457051.020767 & 16.1 & 2.6 & 5.8 & 4.7 & APF \\
\hline 2457051.041243 & 24.1 & 2.7 & -1.1 & 2.8 & APF \\
\hline 2457052.825840 & -11.8 & 2.9 & 7.1 & 7.1 & APF \\
\hline 2457054.832772 & -0.9 & 3.2 & 4.7 & 4.3 & APF \\
\hline
\end{tabular}

(This table is available in its entirety in machine-readable form.)

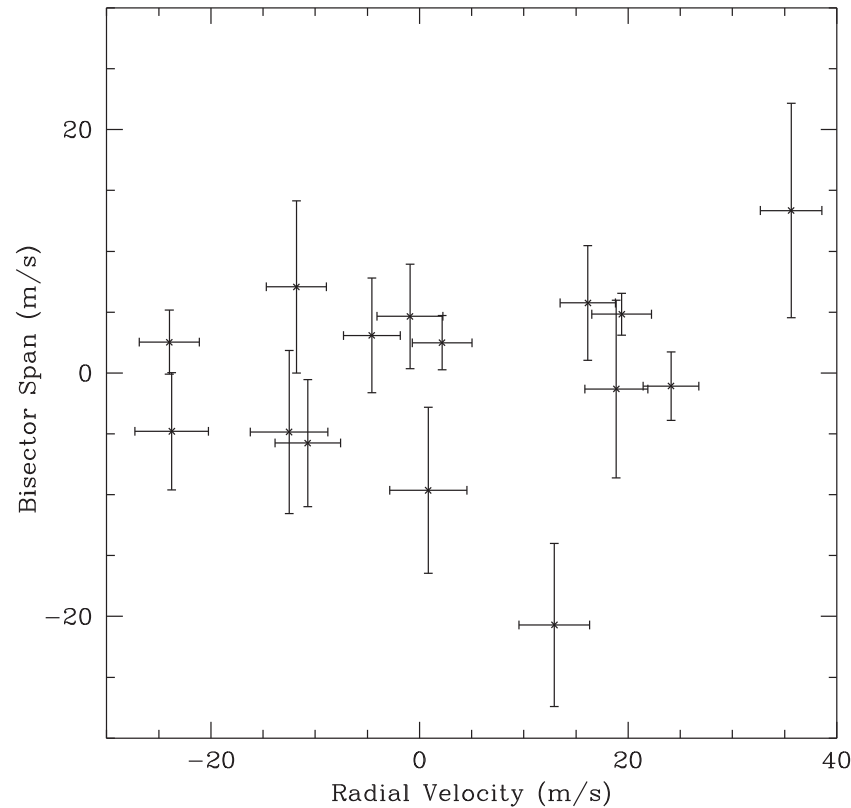

Figure 4. Bisector spans for the APF RV spectra plotted against the RV values. No correlation between the quantities is seen that would indicate that the RV signal is caused by line distortions from an unresolved eclipsing binary instead of true reflex motion of the star KELT- 11.

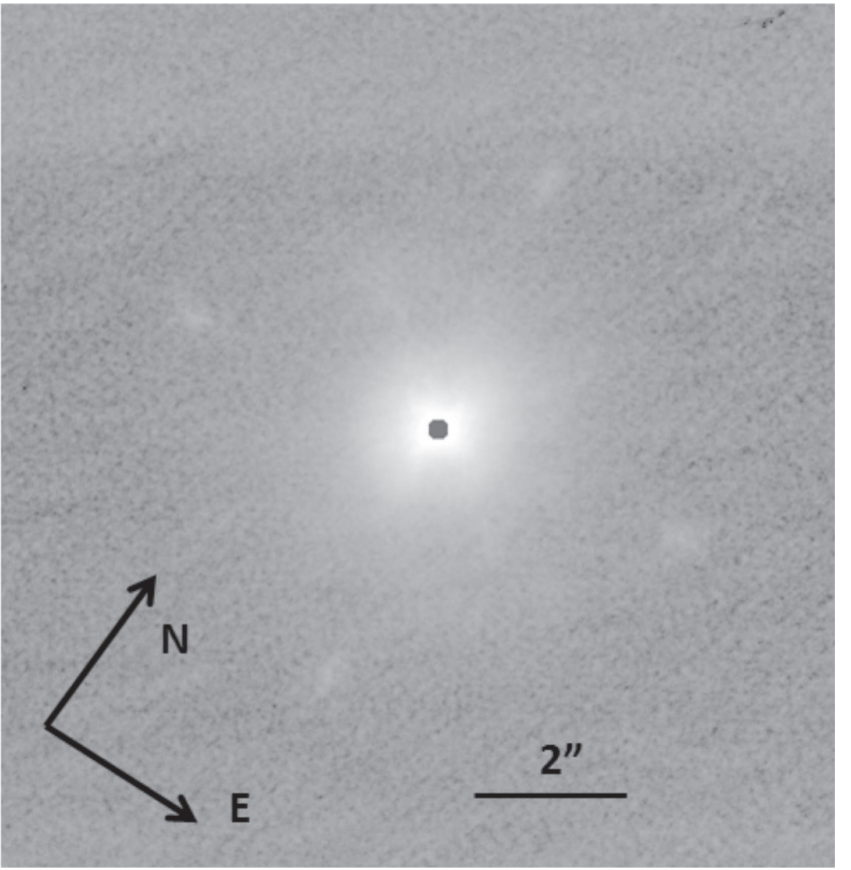

Figure 5. Palomar AO image of KELT-11. The four spots oriented cardinally with the target are a result of the alignment procedure and are not astrophysical. 


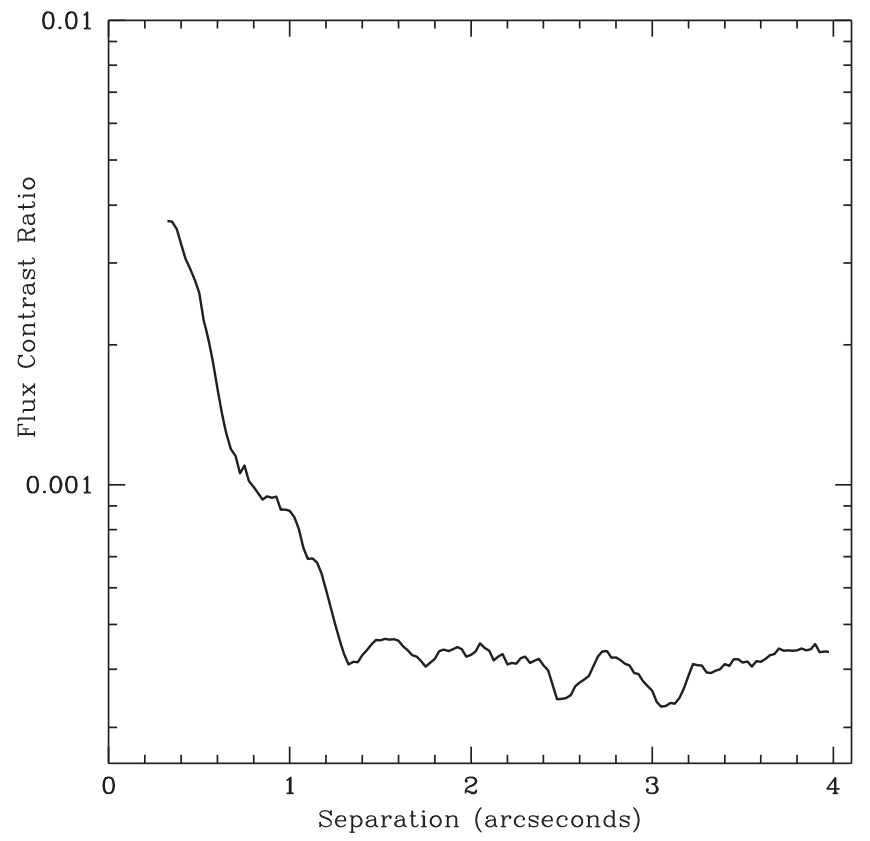

Figure 6. Contrast curve based on Palomar AO imaging. After removing systematics and the point-spread function of the target, no nearby stars are seen within an annulus of radii $\sim 1$ !. $2-4^{\prime \prime}$ down to a flux ratio of $<4 \times 10^{-4}$.

Table 7

Stellar Parameters from the Spectral Analysis

\begin{tabular}{lccc}
\hline \hline Parameter & TRES & KECK HIRES & APF \\
\hline$T_{\text {eff }}(\mathrm{K})$ & $5390 \pm 50$ & $5413 \pm 60$ & $5444 \pm 60$ \\
$\log g_{*}$ & $3.79 \pm 0.10$ & $3.87 \pm 0.08$ & $3.86 \pm 0.08$ \\
{$[\mathrm{Fe} / \mathrm{H}]$} & $0.19 \pm 0.08^{\mathrm{a}}$ & $0.31 \pm 0.04$ & $0.37 \pm 0.04$ \\
\hline
\end{tabular}

Note.

${ }^{a}$ The SME analysis used for the TRES spectra yields a value for bulk metallicity $[m / H]$ rather than $[\mathrm{Fe} / \mathrm{H}]$.

of the host star. The parameter that most strongly controls the duration of the star's time during the subgiant phase is the stellar mass. Therefore, we concentrate our subsequent discussion on our confidence in the accuracy of our estimate of the stellar mass, and in particular our inference that the star is relatively massive $\left(M_{*} \gtrsim 1.3 M_{\odot}\right)$.

The fiducal parameters (including mass) we adopt for KELT11 are obtained using a global fit as described in Section 3.5. Along with the photometric and RV data, this fit used priors on the stellar effective temperature and metallicity from highresolution spectroscopy, and stellar radius as determined from the SED and Hipparcos parallax (see Section 3.2). For our fiducial adopted values, we also used constraints from the YY evolutionary models and assumed zero eccentricity. We also explored the parameters inferred assuming eccentric orbits, as well as using the Torres et al. (2010) empirical relations for the mass and radius of the host star as a function of the stellar effective temperature, surface gravity, and metallicity.

Our procedure, or similar procedures (see Holman et al. 2007; Sozzetti et al. 2007), are commonly used to estimate the masses and radii of transiting planet hosts and their planets. However, it is worth recalling that a single-lined spectroscopic binary with only a primary eclipse does not yield a complete

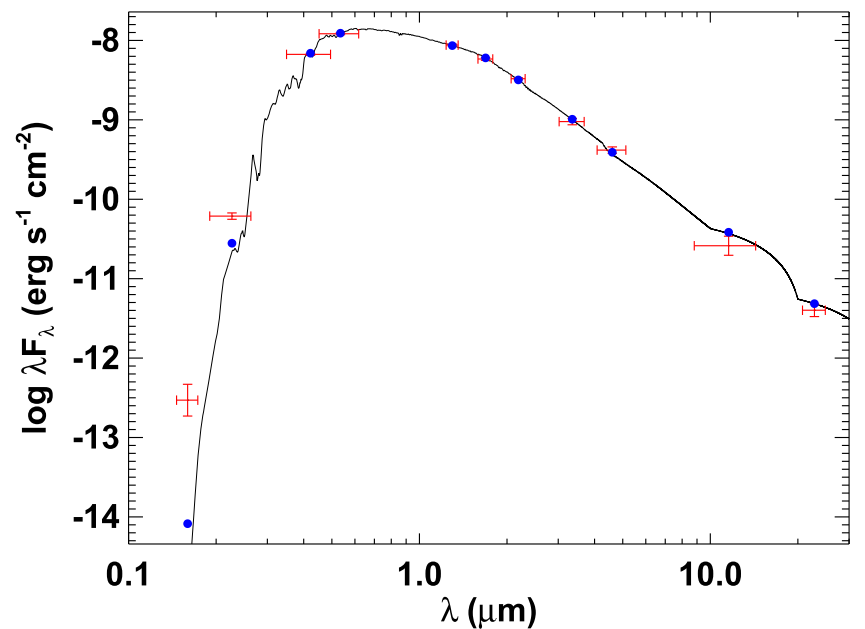

Figure 7. Measured and best-fitting spectral energy distribution (SED) for KELT-11 from ultraviolet through mid-infrared. The red error bars indicate measurements of the flux of KELT-11 in ultraviolet, optical, near-infrared, and mid-infrared passbands as listed in Table 1 . The vertical bars are the $1 \sigma$ photometric uncertainties, whereas the horizontal error bars are the effective widths of the passbands. The solid curve is the best-fitting theoretical SED from the models of Kurucz (1992), assuming stellar parameters $T_{\text {eff }}, \log g_{*}$, and $[\mathrm{Fe} / \mathrm{H}]$ fixed at the adopted values in Tables 8 and 9 , with the extinction and distance $\left(A_{v}\right.$ and $\left.d\right)$ allowed to vary. The blue dots are the predicted passbandintegrated fluxes of the best-fitting theoretical SED corresponding to our observed photometric bands.

solution to the system. Indeed, as pointed out by Seager \& Mallén-Ornelas (2003), the only parameter that is directly measured from the photometry and RVs of such a system is the parameter $a / R_{*}$, which can be related to the stellar density with the (typically reasonable) assumption that $R_{p} \ll R_{*}$ and $M_{p} \ll M_{*}$ (and a measurement or constraint on the eccentricity). Therefore, there is a one-parameter degeneracy between $M_{*}$ and $R_{*}$ for such systems, unless one includes additional direct observables of the system, or invokes model isochrones or empirical relations based on other stars. Using the latter may have some pitfalls, as described below.

For stars with properties that are not too different from the Sun, we can expect that both the YY isochrones and Torres et al. (2010) relations should be fairly reliable. However, it is worth pointing out that it is known that sometimes these do not agree. In particular, Collins et al. (2014) demonstrated that the parameters inferred for the host star of KELT-6b, which happens to be relatively metal-poor $([\mathrm{Fe} / \mathrm{H}] \sim-0.3)$, are significantly different when obtained using the YY constraints relative to those obtained using the Torres et al. (2010) relations. This is likely because the YY isochrones and/or the Torres et al. (2010) relations are not very accurate at low metallicity. It is also worth noting that only a small subset of the stars used to calibrate the Torres et al. (2010) relations have metallicity measurements.

In the case of KELT-11, we are fortunate to have another constraint on the property of the host star, specifically the host star radius. However, there are also some unusual difficulties with this system. Because the parallax measurement is relatively imprecise $(\sim 10 \%)$, this radius constraint is relatively poor. Furthermore, the constraint on the stellar density measured directly from the light curve and the radial velocities is also relatively poor, due to the shallow depth and long duration, and the relatively weak constraint on the eccentricity. 
Table 8

Median Values and 68\% Confidence Interval for the Physical and Orbital Parameters of the KELT-11 System

\begin{tabular}{|c|c|c|c|c|c|}
\hline Parameter & Units & $\begin{array}{l}\text { Adopted Value } \\
\text { (YY Circular; } e=0 \text { Fixed) }\end{array}$ & $\begin{array}{l}\text { Value } \\
\text { (YY Eccentric) }\end{array}$ & $\begin{array}{l}\text { Value } \\
\text { (Torres Circular; } e=0 \\
\text { Fixed) }\end{array}$ & $\begin{array}{l}\text { Value } \\
\text { (Torres Eccentric) }\end{array}$ \\
\hline \multicolumn{6}{|c|}{ Stellar Parameters } \\
\hline$R_{*}$ & Radius $\left(R_{\odot}\right)$ & $2.72_{-0.17}^{+0.21}$ & $2.81_{-0.19}^{+0.21}$ & $2.78_{-0.22}^{+0.24}$ & $2.89 \pm 0.22$ \\
\hline$L_{*}$ & Luminosity $\left(L_{\odot}\right)$ & $5.55_{-0.69}^{+0.85}$ & $5.90_{-0.79}^{+0.86}$ & $5.86_{-0.90}^{+1.1}$ & $6.28_{-0.92}^{+1.0}$ \\
\hline$\rho_{*}^{*}$ & Density (cgs) & $0.101 \pm 0.017$ & $0.093_{-0.015}^{+0.018}$ & $0.088_{-0.017}^{+0.022}$ & $0.080_{-0.014}^{+0.018}$ \\
\hline$T_{\text {eff }}$ & Effective temperature $(\mathrm{K})$ & $5370_{-50}^{+51}$ & $5367 \pm 50$ & $5386 \pm 49$ & $5385_{-50}^{+49}$ \\
\hline$[\mathrm{Fe} / \mathrm{H}]$ & Metallicity & $0.180 \pm 0.075$ & $0.173_{-0.077}^{+0.076}$ & $0.194 \pm 0.079$ & $0.191 \pm 0.079$ \\
\hline \multicolumn{6}{|c|}{ Planet Parameters } \\
\hline$e$ & Eccentricity & $\ldots$ & $0.066_{-0.046}^{+0.059}$ & $\ldots$ & $0.087_{-0.058}^{+0.076}$ \\
\hline$\omega_{*}$ & Argument of periastron $\left(^{\circ}\right)$ & $\cdots$ & $97_{-65}^{+82}$ & $\cdots$ & $86_{-46}^{+69}$ \\
\hline$\rho_{P}$ & Density(cgs) & $0.093_{-0.024}^{+0.028}$ & $0.088_{-0.021}^{+0.027}$ & $0.081_{-0.022}^{+0.030}$ & $0.076_{-0.019}^{+0.025}$ \\
\hline $\log g_{P}$ & Surface gravity & $2.407_{-0.086}^{+0.080}$ & $2.392_{-0.083}^{+0.081}$ & $2.360_{-0.096}^{+0.093}$ & $2.347_{-0.087}^{+0.085}$ \\
\hline$T_{\mathrm{eq}}$ & Equilibrium temperature $(\mathrm{K})$ & $1712_{-46}^{+51}$ & $1733_{-50}^{+49}$ & $1755 \pm 64$ & $1782 \pm 60$ \\
\hline$\Theta^{\mathrm{a}}$ & Safronov number & $0.0123_{-0.0015}^{+0.0016}$ & $0.0120_{-0.0016}^{+0.0017}$ & $0.0119_{-0.0016}^{+0.0017}$ & $0.0117_{-0.0015}^{+0.0017}$ \\
\hline$\langle F\rangle$ & $\begin{array}{l}\text { Incident flux } \\
\quad\left(10^{9} \mathrm{erg} \mathrm{s}^{-1} \mathrm{~cm}^{-2}\right)\end{array}$ & $1.95_{-0.20}^{+0.24}$ & $2.04_{-0.22}^{+0.23}$ & $2.15_{-0.30}^{+0.33}$ & $2.26_{-0.28}^{+0.30}$ \\
\hline \multicolumn{6}{|c|}{ RV Parameters } \\
\hline$T_{C}$ & $\begin{array}{l}\text { Time of inferior conjunc- } \\
\text { tion }\left(\mathrm{BJD}_{\mathrm{TDB}}\right)\end{array}$ & $2457061.9098_{-0.0024}^{+0.0026}$ & $2457061.9098_{-0.0025}^{+0.0027}$ & $2457061.9101_{-0.0025}^{+0.0027}$ & $2457061.9101_{-0.0024}^{+0.0026}$ \\
\hline$T_{P}$ & Time of periastron $\left(\mathrm{BJD}_{\mathrm{TDB}}\right)$ & $\cdots$ & $2457061.99_{-0.74}^{+1.0}$ & $\cdots$ & $2457061.89_{-0.48}^{+0.84}$ \\
\hline$K$ & RV semi-amplitude $\left(\mathrm{m} \mathrm{s}^{-1}\right)$ & $18.5 \pm 1.7$ & $18.7 \pm 1.9$ & $18.5_{-1.6}^{+1.7}$ & $18.8_{-1.9}^{+2.0}$ \\
\hline
\end{tabular}

Note.

${ }^{\mathrm{a}}$ Hansen \& Barman (2007).

Therefore, our final estimate of the mass and radius of KELT11 is strongly influenced by our prior from the YY models or the Torres et al. (2010) relations. This is problematic because the YY models have not been well-calibrated for massive subdwarfs (because there are so few of them with direct mass measurements), and because there are no stars in the Torres et al. (2010) sample with the parameters we infer for KELT-11. Thus by invoking the Torres et al. (2010) relations, we are essentially extrapolating them from where they were calibrated.

Thus, given our relatively a priori unlikely inference that this is a relatively massive star (in a short phase of its lifetime), it is worth exploring what "model-independent" estimates of the stellar mass we can infer. There are two essentially direct (but not entirely independent) methods we can use to estimate the mass of the star without invoking the YY isochrones or the Torres et al. (2010) relations.
First, we can use the estimate of the surface gravity of the star from high-resolution spectra, combined with the measurement of $R_{*}$ from the parallax and SED, to break the $M_{*}-R_{*}$ degeneracy. This is problematic on its own because the surface gravities derived from spectra can have relatively large systemic errors, even with high $\mathrm{S} / \mathrm{N}$ spectra (Torres et al. 2012), and furthermore spectroscopic surface gravities are also notoriously inaccurate for stars on the subgiant branch (Holtzman et al. 2015). Nevertheless, we can employ this process to provide a "direct" method of estimating the mass of KELT-11. Adopting the surface gravity from the TRES spectra of $\log g_{*}=3.79 \pm 0.10$, we find $M_{*}=1.81 \pm 0.44 M_{\odot}$. Adopting instead $\log g_{*}=3.86 \pm 0.10$ from the APF or Keck spectra, we find $M_{*}=2.13 \pm 0.57 M_{\odot}$. Thus, this procedure leads us to infer that KELT-11 is a relatively massive star. The mass we infer is significantly higher and less precise than the 


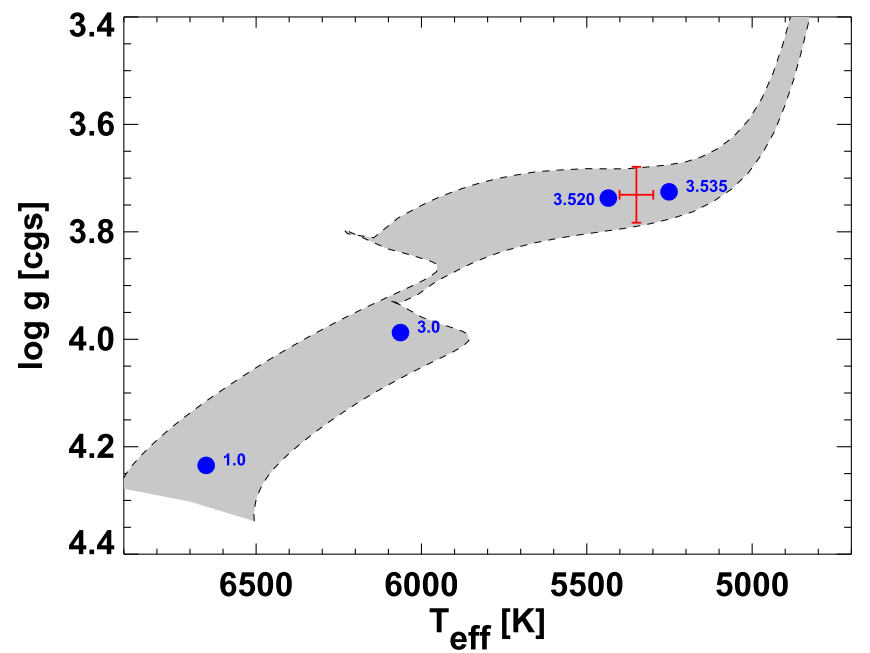

Figure 8. Theoretical H-R diagram for KELT-11b using the Yonsei-Yale stellar evolution models (Demarque et al. 2004). The final global fit values are shown by the red cross. The blue points mark various ages along the tracks. The gray shaded region is a $1 \sigma$ uncertainty on $[\mathrm{Fe} / \mathrm{H}]$ and $M_{\star}$ from the global modeling.

global fit results, but formally does not rely on any models or empirical relations (though it does rely on the spectroscopic surface gravity).

Second, if we wish to avoid using the spectroscopic $\log g_{*}$ as well as any stellar models or empirical relations, we could in principle determine the stellar mass purely through the transit light curve and the stellar radius determined from the parallax and SED. The relation between the stellar mass and the direct observables is

$$
M_{*}=\left(\frac{4 P R_{*}^{3}}{\pi G T_{\mathrm{FWMH}}^{3}}\right)\left(\frac{\sqrt{1-e^{2}}}{1+e \sin \omega}\right)^{3}\left(1-b^{2}\right)^{3 / 2},
$$

assuming $R_{p} \ll R_{*}$ and $M_{p} \ll M_{*}$. The first factor in parentheses is reasonably well measured, and is $\sim 1.82 M_{\odot}$. This depends on our estimate of $T_{\text {FWHM }}$ from the global fit, but this is fairly well constrained from the follow-up photometry alone. We are also in the process of analyzing Spitzer observations (Spitzer ID 12096) of the primary eclipse of this target, and a preliminary analysis verifies the value of $T_{\mathrm{FWHM}}$ derived in this paper. The second factor in parentheses depends on the eccentricity, which is not well constrained. We find that the orbit is consistent with circular, but the eccentricity could be as high as 0.16 at $1 \sigma$. Thus this factor may vary from $\sim 0.5$ to $\sim 1.3$. Finally, the impact parameter is also poorly constrained by our data due the shallowness and duration of the transit, but is constrained to be $<0.5$ at $1 \sigma$. In fact, visual inspect of our preliminary Spitzer reduction indicates that the transit is unlikely to have an impact parameter as high as $\sim 0.5$. Nevertheless, allowing for this full range, we find that the last term can be anywhere between 1 and 0.65 . Thus the full range of allowed masses is $0.6-2.4 M_{\odot}$, although we strongly favor values of $\gtrsim 1.6 M_{\odot}$ based on our preliminary Spitzer data that are consistent with a nearly central transit and our strong prior that the orbit is likely to be circular.

Thus we have four methods of estimating the mass of the primary: (1) using YY isochrones, (2) using the Torres empirical relations, (3) using the spectroscopic $\log g_{*}$ combined with an estimate of the stellar radius from the SED and parallax, and (4) using the density of the star from the transit period and duration, impact parameter, and a constraint on the eccentricity combined with the radius from the SED and parallax. These four methods demonstrate that KELT-11 has a mass around $\gtrsim 1.3 M_{\odot}$, and potentially larger. In particular, the global modeling using the YY isochrones and the Torres et al. (2010) relations finds good agreement, deriving masses of $M_{*}=1.438_{-0.052}^{+0.061} M_{\odot}$ and $M_{*}=1.347_{-0.068}^{+0.072} M_{\odot}$, respectively (assuming a circular orbit). The model-independent or quasimodel-independent methods described above yield larger mass estimates but with significantly larger errors, and thus all four estimates are nominally consistent. Although it is possible (and perhaps even likely) that there are significant systematic errors in all four of these estimates, we believe it is unlikely that those sytematics would all collude to lead us to the interpretation that this star is significantly more massive than the Sun, if, in fact, it is not.

The fact that KELT-11 hosts a transiting planet makes it a particularly valuable member of the "Retired A-star" sample of Johnson et al. (2007). Stars with transiting planets allow an additional constraint on the properties of the host star, namely a direct measurement of $a / R_{*}$, and, given the (reasonable) assumption of $R_{p} \ll R_{*}$ and $M_{p} \ll M_{*}$, and a measurement or constraint on the eccentricity, and an estimate of the host star density $\rho_{*}$. Currently, the constraint on the density of KELT-11 is relatively poor because of the imprecise measurement of transit duration and ingress/egress time $\left(T_{\mathrm{FWHM}}\right.$ and $\tau$ ) due to the relatively shallow and long duration of the transit. Nevertheless, when combined with an independent estimate of its radius derived from the fact that it has a direct distance measurement via the Hipparcos parallax, this provides a second, purely empirical estimate of the mass of the host star. The fact that this estimate agrees with our more modeldependent estimate of the host star mass via global fitting using isochrones, and the fact that these further agree with our estimate of the host star using the empirical relations of Torres et al. (2010), gives us confidence that the host star mass is indeed significantly more massive than $\sim 1.2 M_{\odot}$, and thus the host star really is a "Retired A (or early F) star."

Once we have fully analyzed the above-mentioned Spitzer follow-up photometry of a primary transit of KELT-11b, we will have a much more precise measurement of $T_{\mathrm{FWHM}}$ and $\tau$, and thus $\rho_{*}$. When combined with an exquisite measurement of the stellar parallax with Gaia (Perryman et al. 2001) and thus stellar radius, we will be able to provide much more accurate, precise, and essentially direct empirical constraint on the host star mass, using the methods described previously.

\section{Summary and Conclusions}

KELT-11b is an extremely inflated planet (Figure 10), with a density of just $0.093_{-0.024}^{+0.028} \mathrm{~g} \mathrm{~cm}^{-3}$. This makes KELT-11b the third lowest-density planet ever discovered with a precisely measured mass and radius (those with parameter uncertainties $<20 \%$ ). The only comparable planets are WASP-94Ab (Neveu-VanMalle et al. 2014) and Kepler-12b (Fortney et al. 2011), but they both orbit significantly fainter hosts.

Another way of placing this planet in context is to note that currently there are only a handful of hot Jupiters that transit bright stars (Figure 10). Of these, KELT-11b has by far the largest atmospheric scale height, at $2763 \mathrm{~km}$, assuming uniform heat redistribution and calculating the scale height along the 
Table 9

Median Values and 68\% Confidence Interval for the Physical and Orbital Parameters of the KELT-11 System

\begin{tabular}{|c|c|c|c|c|c|}
\hline Parameter & Units & $\begin{array}{l}\text { Adopted Value } \\
\text { (YY Circular; } e=0 \text { Fixed) }\end{array}$ & $\begin{array}{l}\text { Value } \\
\text { (YY Eccentric) }\end{array}$ & $\begin{array}{l}\text { Value } \\
\text { (Torres Circular; } e=0 \\
\text { Fixed) }\end{array}$ & $\begin{array}{l}\text { Value } \\
\text { (Torres Eccentric) }\end{array}$ \\
\hline \multicolumn{6}{|c|}{ Primary Transit } \\
\hline$R_{P} / R_{*}$ & $\begin{array}{l}\text { Radius of the planet in stellar } \\
\text { radii }\end{array}$ & $0.0519 \pm 0.0026$ & $0.0516 \pm 0.0026$ & $0.0526 \pm 0.0027$ & $0.0520 \pm 0.0026$ \\
\hline$a / R_{*}$ & Semimajor axis in stellar radii & $4.93_{-0.29}^{+0.26}$ & $4.80_{-0.27}^{+0.28}$ & $4.71_{-0.32}^{+0.36}$ & $4.56_{-0.29}^{+0.32}$ \\
\hline$i$ & Inclination $\left(^{\circ}\right)$ & $85.8_{-1.8}^{+2.4}$ & $86.4_{-2.2}^{+2.3}$ & $84.4_{-1.8}^{+2.6}$ & $85.4_{-2.3}^{+2.9}$ \\
\hline$b$ & Impact parameter & $0.36_{-0.20}^{+0.13}$ & $0.29_{-0.19}^{+0.17}$ & $0.46_{-0.19}^{+0.11}$ & $0.34_{-0.22}^{+0.17}$ \\
\hline$\delta$ & Transit depth & $0.00269_{-0.00026}^{+0.00028}$ & $0.00267_{-0.00026}^{+0.00027}$ & $0.00276_{-0.00028}^{+0.00029}$ & $0.00271_{-0.00027}^{+0.00028}$ \\
\hline$T_{\text {FWHM }}$ & FWHM duration (days) & $0.2874 \pm 0.0048$ & $0.2873 \pm 0.0047$ & $0.2876_{-0.0048}^{+0.0049}$ & $0.2877_{-0.0045}^{+0.0048}$ \\
\hline$\tau$ & Ingress/egress duration (days) & $0.0173_{-0.0021}^{+0.0029}$ & $0.0164_{-0.0016}^{+0.0029}$ & $0.0193_{-0.0032}^{+0.0038}$ & $0.0172_{-0.0021}^{+0.0039}$ \\
\hline$T_{14}$ & Total duration (days) & $0.3051_{-0.0051}^{+0.0053}$ & $0.3043_{-0.0052}^{+0.0053}$ & $0.3072_{-0.0053}^{+0.0059}$ & $0.3056_{-0.0052}^{+0.0058}$ \\
\hline$P_{T}$ & $\begin{array}{l}\text { A priori non-grazing transit } \\
\text { probability }\end{array}$ & $0.1925_{-0.0096}^{+0.012}$ & $0.205_{-0.018}^{+0.024}$ & $0.201 \pm 0.014$ & $0.221_{-0.024}^{+0.033}$ \\
\hline$P_{T, G}$ & A priori transit probability & $0.214_{-0.011}^{+0.013}$ & $0.228_{-0.020}^{+0.027}$ & $0.224_{-0.016}^{+0.017}$ & $0.246_{-0.027}^{+0.037}$ \\
\hline$T_{C, 0}$ & Mid-transit time $\left(\mathrm{BJD}_{\mathrm{TDB}}\right)$ & $2457024.0176_{-0.0028}^{+0.0030}$ & $2457024.0175_{-0.0029}^{+0.0030}$ & $2457024.0179_{-0.0029}^{+0.0031}$ & $2457024.0179_{-0.0027}^{+0.0030}$ \\
\hline$T_{C, 1}$ & Mid- transit time $\left(\mathrm{BJD}_{\mathrm{TDB}}\right)$ & $2457061.9098_{-0.0024}^{+0.0026}$ & $2457061.9098_{-0.0025}^{+0.0027}$ & $2457061.9101_{-0.0025}^{+0.0027}$ & $2457061.9101_{-0.0024}^{+0.0026}$ \\
\hline$T_{C, 2}$ & Mid-transit time $\left(\mathrm{BJD}_{\mathrm{TDB}}\right)$ & $2457061.9098_{-0.0024}^{+0.0026}$ & $2457061.9098_{-0.0025}^{+0.0027}$ & $2457061.9101_{-0.0025}^{+0.0027}$ & $2457061.9101_{-0.0024}^{+0.0026}$ \\
\hline$T_{C, 3}$ & Mid-transit time $\left(\mathrm{BJD}_{\mathrm{TDB}}\right)$ & $2457061.9098_{-0.0024}^{+0.0026}$ & $2457061.9098_{-0.0025}^{+0.0027}$ & $2457061.9101_{-0.0025}^{+0.0027}$ & $2457061.9101_{-0.0024}^{+0.0026}$ \\
\hline$T_{C, 4}$ & Mid-transit time $\left(\mathrm{BJD}_{\mathrm{TDB}}\right)$ & $2457090.3290_{-0.0022}^{+0.0024}$ & $2457090.3289_{-0.0022}^{+0.0024}$ & $2457090.3293_{-0.0023}^{+0.0025}$ & $2457090.3292_{-0.0022}^{+0.0024}$ \\
\hline$T_{C, 5}$ & Mid-transit time $\left(\mathrm{BJD}_{\mathrm{TDB}}\right)$ & $2457095.0655_{-0.0022}^{+0.0024}$ & $2457095.0655_{-0.0022}^{+0.0024}$ & $2457095.0658_{-0.0022}^{+0.0024}$ & $2457095.0657_{-0.0022}^{+0.0024}$ \\
\hline$T_{C, 6}$ & Mid- transit time $\left(\mathrm{BJD}_{\mathrm{TDB}}\right)$ & $2457099.8021_{-0.0021}^{+0.0024}$ & $2457099.8020_{-0.0022}^{+0.0024}$ & $2457099.8024_{-0.0022}^{+0.0024}$ & $2457099.8023_{-0.0021}^{+0.0024}$ \\
\hline$T_{C, 7}$ & Mid-transit time $\left(\mathrm{BJD}_{\mathrm{TDB}}\right)$ & $2457147.1674_{-0.0019}^{+0.0021}$ & $2457147.1673_{-0.0020}^{+0.0021}$ & $2457147.1677_{-0.0019}^{+0.0021}$ & $2457147.1675_{-0.0019}^{+0.0021}$ \\
\hline$T_{C, 8}$ & Mid-transit time $\left(\mathrm{BJD}_{\mathrm{TDB}}\right)$ & $2457440.8323_{-0.0036}^{+0.0042}$ & $2457440.8319_{-0.0036}^{+0.0041}$ & $2457440.8325_{-0.0038}^{+0.0043}$ & $2457440.8318_{-0.0037}^{+0.0040}$ \\
\hline$u_{1 I}$ & Linear Limb-darkening & $0.3466_{-0.0093}^{+0.0094}$ & $0.3462_{-0.0092}^{+0.0094}$ & $0.3437 \pm 0.0094$ & $0.3434 \pm 0.0094$ \\
\hline$u_{2 I}$ & Quadratic Limb-darkening & $0.2510 \pm 0.0050$ & $0.2511 \pm 0.0049$ & $0.2533 \pm 0.0050$ & $0.2536 \pm 0.0050$ \\
\hline$u_{1 R}$ & Linear Limb-darkening & $0.446 \pm 0.012$ & $0.446 \pm 0.012$ & $0.443 \pm 0.012$ & $0.443 \pm 0.012$ \\
\hline$u_{2 R}$ & Quadratic Limb-darkening & $0.2374_{-0.0071}^{+0.0070}$ & $0.2376 \pm 0.0070$ & $0.2398_{-0.0071}^{+0.0070}$ & $0.2401_{-0.0070}^{+0.0071}$ \\
\hline$u_{1 \text { Sloang }}$ & Linear Limb-darkening & $0.695 \pm 0.017$ & $0.695 \pm 0.017$ & $0.692 \pm 0.017$ & $0.692 \pm 0.017$ \\
\hline$u_{2 \text { Sloang }}$ & Quadratic Limb-darkening & $0.117 \pm 0.013$ & $0.117 \pm 0.013$ & $0.120 \pm 0.013$ & $0.120 \pm 0.013$ \\
\hline$u_{1 \text { Sloani }}$ & Linear Limb-darkening & $0.371 \pm 0.010$ & $0.3702_{-0.0100}^{+0.010}$ & $0.368 \pm 0.010$ & $0.367 \pm 0.010$ \\
\hline$u_{2 \text { Sloani }}$ & Quadratic Limb-darkening & $0.2485_{-0.0055}^{+0.0054}$ & $0.2486 \pm 0.0054$ & $0.2508 \pm 0.0055$ & $0.2511_{-0.0055}^{+0.0054}$ \\
\hline$u_{1 \text { Sloanr }}$ & Linear Limb-darkening & $0.476 \pm 0.013$ & $0.475 \pm 0.013$ & $0.472 \pm 0.013$ & $0.472 \pm 0.013$ \\
\hline$u_{2 \text { Sloanr }}$ & Quadratic Limb-darkening & $0.2316_{-0.0078}^{+0.0077}$ & $0.2318_{-0.0077}^{+0.0076}$ & $0.2340 \pm 0.0077$ & $0.2343 \pm 0.0077$ \\
\hline$u_{1 \text { Sloanz }}$ & Linear Limb-darkening & $0.2976 \pm 0.0075$ & $0.2974 \pm 0.0074$ & $0.2952_{-0.0075}^{+0.0074}$ & $0.2949 \pm 0.0075$ \\
\hline$u_{2 \text { Sloanz }}$ & Quadratic Limb-darkening & $0.2549_{-0.0036}^{+0.0037}$ & $0.2549_{-0.0036}^{+0.0035}$ & $0.2568 \pm 0.0036$ & $0.2570 \pm 0.0036$ \\
\hline \multicolumn{6}{|l|}{$\begin{array}{l}\text { Secondary } \\
\text { Eclipse }\end{array}$} \\
\hline$T_{S}$ & Time of eclipse $\left(\mathrm{BJD}_{\mathrm{TDB}}\right)$ & $2457059.5415_{-0.0025}^{+0.0027}$ & $2457064.27_{-0.16}^{+0.15}$ & $2457059.5418_{-0.0025}^{+0.0027}$ & $2457064.28_{-0.15}^{+0.19}$ \\
\hline$b_{S}$ & Impact parameter & $\ldots$ & $0.31_{-0.20}^{+0.16}$ & $\ldots$ & $0.39_{-0.24}^{+0.15}$ \\
\hline$T_{S, \text { FWHM }}$ & FWHM duration (days) & $\ldots$ & $0.303_{-0.022}^{+0.042}$ & $\cdots$ & $0.315_{-0.029}^{+0.057}$ \\
\hline$\tau_{S}$ & Ingress/egress duration (days) & $\ldots$ & $0.0183_{-0.0024}^{+0.0029}$ & $\ldots$ & $0.0205_{-0.0031}^{+0.0039}$ \\
\hline$T_{S, 14}$ & Total duration (days) & $\cdots$ & $0.322_{-0.023}^{+0.043}$ & $\cdots$ & $0.336_{-0.030}^{+0.058}$ \\
\hline$P_{S}$ & $\begin{array}{l}\text { A priori non-grazing eclipse } \\
\text { probability }\end{array}$ & $\ldots$ & $0.1890_{-0.0069}^{+0.013}$ & $\ldots$ & $0.1934_{-0.0093}^{+0.016}$ \\
\hline$P_{S, G}$ & A priori eclipse probability & $\ldots$ & $0.2095_{-0.0079}^{+0.014}$ & $\ldots$ & $0.215_{-0.011}^{+0.018}$ \\
\hline
\end{tabular}

lines of Winn (2010, pp. 55-77). The ratio of scale height to planet radius is $2.8 \%$, with an expected size of the signal from transmission spectroscopy of 5.6\%, making KELT-11b particularly amenable to atmospheric characterization via transmission or emission spectroscopy. The expected depth of the secondary eclipse in $K$ is $1.4 \times 10^{-4}$, assuming the planetary dayside is at a blackbody equilibrium temperature of $1710 \mathrm{~K}$.

Ultimately, the bright host star, the inflated radius, and the high equilibrium temperature make KELT-11b one of the best targets discovered to date for transmission spectroscopy. For example, detailed studies of KELT-11b will allow its chemical composition to be determined, which in turn will constrain parameters involving its formation and evolution of planets (e.g., Madhusudhan et al. 2014). Furthermore, the source of inflation in hot Jupiters can be investigated in the extreme case of KELT-11b. Future observations of KELT-11b with facilities like The Hubble Space Telescope, Spitzer, and JWST will reveal the structure and content of its atmosphere, and will set up KELT-11b as the benchmark sub-Saturn exoplanet.

It is also noteworthy that this planet has the shallowest transit depth (2.69 mmag) of any planet discovered by a ground-based transit survey, with the next-shallowest such planet having a 


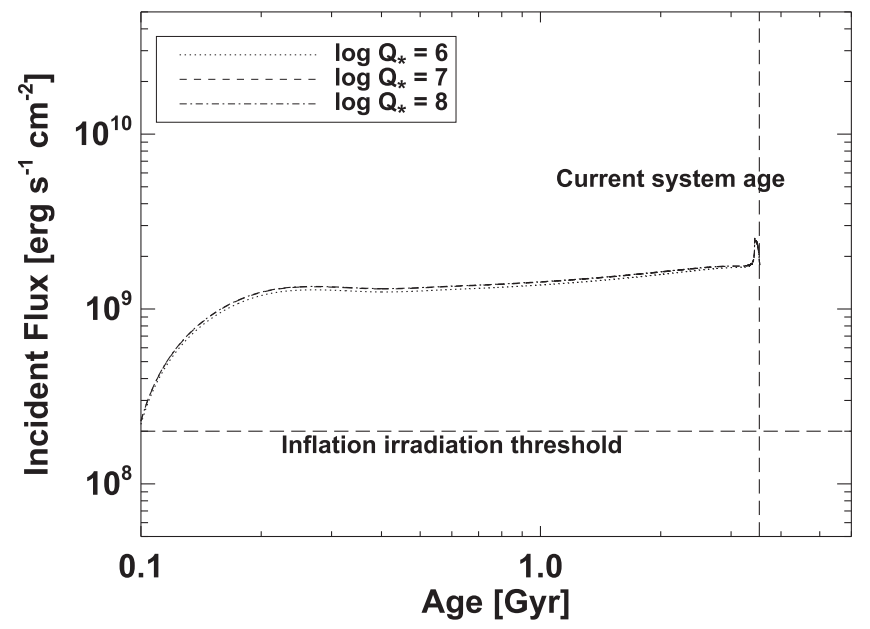

Figure 9. History of the inflation irradiation for KELT-11 for test values of $\log Q_{\star}^{\prime}$ from six to eight. The horizontal dashed line indicates the insolation threshold determined by Demory \& Seager (2011).

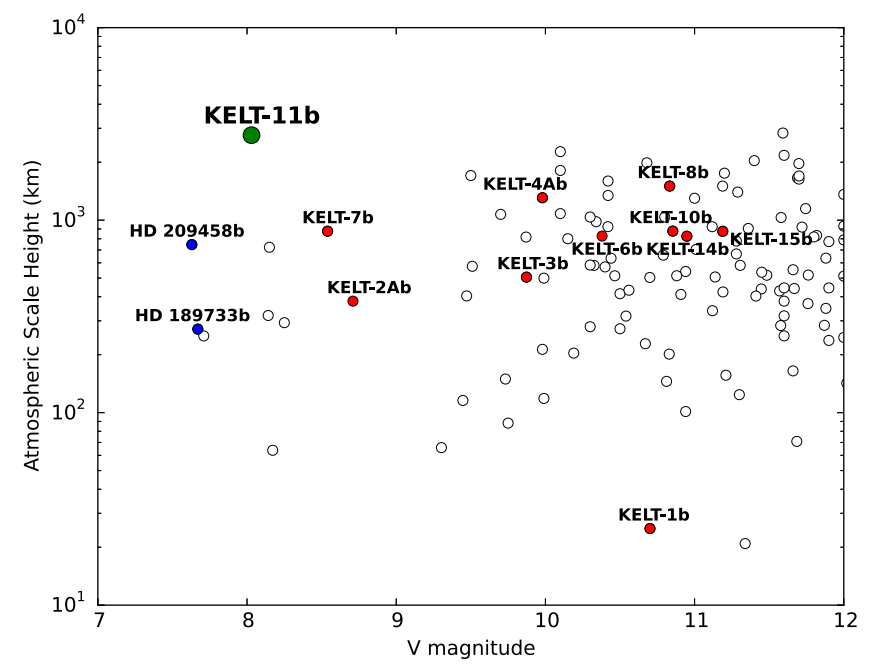

Figure 10. Estimated atmospheric scale height of known transiting hot Jupiters vs. the $V$-band brightness of the host star. KELT- $11 \mathrm{~b}$ is highlighted by a filled green circle, while other discoveries from the KELT survey are marked by filled red circles. The filled blue circles mark the two well-studied benchmark hot Jupiters HD 209458b and HD 189733b. The open circles mark other known transiting exoplanets from the NASA Exoplanet Archive (accessed on 2016 May 28).

transit depth of 3.3 mmag (HAT-P-11b; Bakos et al. 2010). Surveys like HAT, KELT, and SuperWASP are still increasing their photometric precision, and, although the TESS survey will provide higher photometric precision over the entire sky, the long time baselines of the ground-based surveys with highquality photometry can help confirm planets with periods longer than the duration of the TESS observations.

As described in Section 3.3, the KELT-11 system exists in a very brief range of the host star's evolution. The star has exhausted its core hydrogen and is contracting such that it is about to begin (or maybe already is) undergoing shell hydrogen fusion. This stage is very short lived (roughly $60 \mathrm{Myr}$ ), and since transiting planets are already rare, finding one with a host star in such a stage is quite fortunate. Once KELT-11 reaches the base of the giant branch, it will engulf KELT-11b, possibly producing a spectacular transient signal (Metzger et al. 2012). Thus, the detection of this one system (because it occupies such a special and short-lived period in the evolution of the star), provides an example of a direct precursor to such planetengulfment events and transients. It also provides an estimate of the frequency of such transient events, which can be used as a prediction for, e.g., LSST. The recent discovery of another transiting giant planet around a subgiant star K2-39b is an additional contribution to this small sample (Van Eylen et al. 2016).

In addition to the potential value of KELT-11b, for characterization of exoatmospheres and the frequency of planets orbiting higher-mass stars, this discovery illustrates certain aspects of the current state of transit discovery. This planet was discovered due to the combination of both transit and RV survey data. The KELT survey observations (Section 2.1) and follow-up photometry (Section 2.2) enabled us to identify this target as a good candidate, but with such a low-mass planet, our typical follow-up methods to obtain an RV orbit would have been extremely hard-pressed to enable dynamical confirmation purely through follow-up RV observations. However, the addition of the CPS survey data provided the evidence that this was a real planet, prompting us to gather the additional APF observations to enable reliable confirmation. Furthermore, the CPS RV observations by themselves were not sufficient to verify HD 93396 as a planet host without the accompanying transit evidence from KELT. We believe that this synergy between multiple types of survey data will be of great value over the next several years, especially with the expected launch of the TESS mission and availability of nearly all-sky high precision photometry.

Work by B.S.G. and D.J.S. was partially supported by NSF CAREER Grant AST-1056524. M.B. is supported by a NASA Space Technology Research Fellowship. J.P. would like to thank Stephen Faulkner and Katheryn Kirkwood for help during a difficult time. D.W.L acknowledges partial support from the Kepler Extended Mission under Cooperative Agreement NNX13AB58A with the Smithsonian Astrophysical Observatory. D.B. acknowledges financial support from the National Centre for Competence in Research PlanetS supported by the Swiss National Science Foundation. T.B. was partially supported by funding from the Center for Exoplanets and Habitable Worlds. The Center for Exoplanets and Habitable Worlds is supported by the Pennsylvania State University, the Eberly College of Science, and the Pennsylvania Space Grant Consortium.

B.J.F. notes that this material is based upon work supported by the National Science Foundation Graduate Research Fellowship under grant No. 2014184874. Any opinion, findings, and conclusions or recommendations expressed in this material are those of the authors(s) and do not necessarily reflect the views of the National Science Foundation.

This work has made use of NASAs Astrophysics Data System, the Extrasolar Planet Encyclopedia at exoplanet.eu (Schneider et al. 2011), the SIMBAD database operated at CDS, Strasbourg, France, and the VizieR catalog access tool, CDS, Strasbourg, France (Ochsenbein et al. 2000).

Certain calculations in this paper were carried out on the Ruby cluster operated by the Ohio Supercomputer Center (Center 1987).

MINERVA is a collaboration among the Harvard-Smithsonian Center for Astrophysics, The Pennsylvania State University, University of Montana, and University of New South Wales. MINERVA is made possible by generous contributions 
from its collaborating institutions and Mt. Cuba Astronomical Foundation, The David \& Lucile Packard Foundation, National Aeronautics and Space Administration (EPSCOR grant NNX13AM97A), and The Australian Research Council (LIEF grant LE140100050).

This publication makes use of data products from the Widefield Infrared Survey Explorer, which is a joint project of the University of California, Los Angeles, and the Jet Propulsion Laboratory/California Institute of Technology, funded by the National Aeronautics and Space Administration.

This publication makes use of data products from the Two Micron All Sky Survey, which is a joint project of the University of Massachusetts and the Infrared Processing and Analysis Center/California Institute of Technology, funded by the National Aeronautics and Space Administration and the National Science Foundation.

This paper makes use of data from the first public release of the WASP data (Butters et al. 2010) as provided by the WASP consortium and services at the NASA Exoplanet Archive (Akeson et al. 2013), which is operated by the California Institute of Technology, under contract with the National Aeronautics and Space Administration under the Exoplanet Exploration Program, the Exoplanet Orbit Database, and the Exoplanet Data Explorer at exoplanets.org (Han et al. 2014).

\section{References}

Akeson, R. L., Chen, X., Ciardi, D., et al. 2013, PASP, 125, 989 Alonso, R., Brown, T. M., Torres, G., et al. 2004, ApJL, 613, L153 Assef, R. J., Gaudi, B. S., \& Stanek, K. Z. 2009, ApJ, 701, 1616 Bakos, G., Noyes, R. W., Kovács, G., et al. 2004, PASP, 116, 266 Bensby, T., Feltzing, S., \& Lundström, I. 2003, A\&A, 410, 527 Borucki, W. J., Koch, D., Basri, G., et al. 2010, Sci, 327, 977 Bottom, M., Kuhn, J., Mennesson, B., et al. 2015, ApJ, 809, 11 Buchhave, L. A., Bakos, G. Á., Hartman, J. D., et al. 2010, ApJ, 720, 1118 Buchhave, L. A., Latham, D. W., Johansen, A., et al. 2012, Natur, 486, 375 Burke, C. J., Gaudi, B. S., DePoy, D. L., \& Pogge, R. W. 2006, AJ, 132, 210 Butler, R. P., Marcy, G. W., Williams, E., et al. 1996, PASP, 108, 500 Butters, O. W., West, R. G., Anderson, D. R., et al. 2010, A\&A, 520, L10

Center, O. S. 1987, Ohio Supercomputer Center, http://osc.edu/ark:/19495/ f5s $1 \mathrm{ph} 73$

Charbonneau, D., Brown, T. M., Latham, D. W., \& Mayor, M. 2000, ApJL, 529, L45

Coşkunoğlu, B., Ak, S., Bilir, S., et al. 2011, MNRAS, 412, 1237

Coelho, P. R. T. 2014, MNRAS, 440, 1027

Collins, K., \& Kielkopf, J. 2013, AstroImageJ: ImageJ for Astronomy, Astrophysics Source Code Library, ascl:1309.001

Collins, K. A., Eastman, J. D., Beatty, T. G., et al. 2014, AJ, 147, 39

Collins, K. A., Kielkopf, J. F., Stassun, K. G., \& Hessman, F. V. 2017, AJ, 153, 77

Cutri, R. M., Skrutskie, M. F., van Dyk, S., et al. 2003, yCat, 2246, 0

Cutri, R. M., et al. 2014, yCat, 2328, 0

Demarque, P., Woo, J.-H., Kim, Y.-C., \& Yi, S. K. 2004, ApJS, 155, 667

Demory, B.-O., \& Seager, S. 2011, ApJS, 197, 12

Eastman, J., Gaudi, B. S., \& Agol, E. 2013, PASP, 125, 83

Fortney, J. J., Demory, B.-O., Désert, J.-M., et al. 2011, ApJS, 197, 9

Fressin, F., Guillot, T., Morello, V., \& Pont, F. 2007, A\&A, 475, 729

Fulton, B. J., Collins, K. A., Gaudi, B. S., et al. 2015a, ApJ, 810, 30

Fulton, B. J., Howard, A. W., Winn, J. N., et al. 2013, ApJ, 772, 80

Fulton, B. J., Weiss, L. M., Sinukoff, E., et al. 2015b, ApJ, 805, 175

Gaudi, B. S. 2005, ApJL, 628, L73

Han, E., Wang, S. X., Wright, J. T., et al. 2014, PASP, 126, 827

Hansen, B. M. S., \& Barman, T. 2007, ApJ, 671, 861

Hartman, J. D., \& Bakos, G. Á. 2016, A\&C, 17, 1
Henry, G. W., Marcy, G. W., Butler, R. P., \& Vogt, S. S. 2000, ApJL, 529, L41 Høg, E., Fabricius, C., Makarov, V. V., et al. 2000, A\&A, 355, L27

Holman, M. J., Winn, J. N., Latham, D. W., et al. 2007, ApJ, 664, 1185 Holtzman, J. A., Shetrone, M., Johnson, J. A., et al. 2015, AJ, 150, 148 Houk, N., \& Swift, C. 1999, in Michigan Spectral Survey, Vol. 5 (Ann Arbor, MI: Dep. Astron. Univ. Michigan), 0

Howard, A. W., Johnson, J. A., Marcy, G. W., et al. 2010, ApJ, 721, 1467

Huber, D., Ireland, M. J., Bedding, T. R., et al. 2012, ApJ, 760, 32

Jenkins, J. S., Murgas, F., Rojo, P., et al. 2011, A\&A, 531, A8

Jensen, E. 2013, Tapir: A Web Interface for Transit/Eclipse Observability, Astrophysics Source Code Library, ascl:1306.007

Johnson, J. A., Clanton, C., Howard, A. W., et al. 2011, ApJS, 197, 26

Johnson, J. A., Fischer, D. A., Marcy, G. W., et al. 2007, ApJ, 665, 785

Johnson, J. A., Howard, A. W., Marcy, G. W., et al. 2010, PASP, 122, 149

Johnson, J. A., Marcy, G. W., Fischer, D. A., et al. 2006, ApJ, 652, 1724

Kibrick, R. I., Clarke, D. A., Deich, W. T. S., \& Tucker, D. 2006, Proc. SPIE, 6274,1

Konacki, M., Torres, G., Jha, S., \& Sasselov, D. D. 2003, Natur, 421, 507

Kovács, G., Zucker, S., \& Mazeh, T. 2002, A\&A, 391, 369

Kuhn, R. B., Rodriguez, J. E., Collins, K. A., et al. 2016, MNRAS, 459, 4281

Kurucz, R. L. 1979, ApJS, 40, 1

Kurucz, R. L. 1992, in IAU Symp. 149, The Stellar Populations of Galaxies, ed. B. Barbuy \& A. Renzini (Cambridge: Cambridge Univ. Press), 225

Madhusudhan, N., Amin, M. A., \& Kennedy, G. M. 2014, ApJL, 794, L12

McCullough, P. R., Stys, J. E., Valenti, J. A., et al. 2006, ApJ, 648, 1228

Metzger, B. D., Giannios, D., \& Spiegel, D. S. 2012, MNRAS, 425, 2778

Neveu-VanMalle, M., Queloz, D., Anderson, D. R., et al. 2014, A\&A, 572, A49

Ochsenbein, F., Bauer, P., \& Marcout, J. 2000, A\&AS, 143, 23

Penev, K., Zhang, M., \& Jackson, B. 2014, PASP, 126, 553

Pepper, J., \& Gaudi, B. S. 2005, ApJ, 631, 581

Pepper, J., Gould, A., \& Depoy, D. L. 2003, AcA, 53, 213

Pepper, J., Kuhn, R. B., Siverd, R., James, D., \& Stassun, K. 2012, PASP, 124,230

Pepper, J., Pogge, R. W., DePoy, D. L., et al. 2007, PASP, 119, 923

Perryman, M. A. C., de Boer, K. S., Gilmore, G., et al. 2001, A\&A, 369, 339

Perryman, M. A. C., Lindegren, L., Kovalevsky, J., et al. 1997, A\&A, 323, 49

Petigura, E. A., Schlieder, J. E., Crossfield, I. J. M., et al. 2015, ApJ, 811, 102

Pollacco, D. L., Skillen, I., Collier Cameron, A., et al. 2006, PASP, 118, 1407

Pont, F., Zucker, S., \& Queloz, D. 2006, MNRAS, 373, 231

Ricker, G. R., Winn, J. N., Vanderspek, R., et al. 2015, JATIS, 1, 014003

Rodriguez, J. E., Colón, K. D., Stassun, K. G., et al. 2016, AJ, 151, 138

Rouan, D., Baglin, A., Copet, E., et al. 1998, EM\&P, 81, 79

Schlegel, D. J., Finkbeiner, D. P., \& Davis, M. 1998, ApJ, 500, 525

Schneider, J., Dedieu, C., Le Sidaner, P., Savalle, R., \& Zolotukhin, I. 2011, A\&A, 532, A79

Seager, S., \& Deming, D. 2010, ARA\&A, 48, 631

Seager, S., \& Mallén-Ornelas, G. 2003, in ASP Conf. Ser. 294, Scientific Frontiers in Research on Extrasolar Planets, ed. D. Deming \& S. Seager (San Francisco, CA: ASP), 419

Sing, D. K., Fortney, J. J., Nikolov, N., et al. 2016, Natur, 529, 59

Siverd, R. J., Beatty, T. G., Pepper, J., et al. 2012, ApJ, 761, 123

Skrutskie, M. F., Cutri, R. M., Stiening, R., et al. 2006, AJ, 131, 1163

Sozzetti, A., Torres, G., Charbonneau, D., et al. 2007, ApJ, 664, 1190

Spiegel, D. S., \& Madhusudhan, N. 2012, ApJ, 756, 132

Swift, J. J., Bottom, M., Johnson, J. A., et al. 2015, JATIS, 1, 027002

Torres, G., Andersen, J., \& Giménez, A. 2010, A\&ARv, 18, 67

Torres, G., Fischer, D. A., Sozzetti, A., et al. 2012, ApJ, 757, 161

Bakos, G. Á., Torres, G., Pál, A., et al. 2010, ApJ, 710, 1724

Udalski, A., Zebrun, K., Szymanski, M., et al. 2002, AcA, 52, 115

Valenti, J. A., \& Fischer, D. A. 2005, ApJS, 159, 141

Van Eylen, V., Albrecht, S., Gandolfi, D., et al. 2016, AJ, 152, 143

van Leeuwen, F. 2007, A\&A, 474, 653

Vogt, S. S., Allen, S. L., Bigelow, B. C., et al. 1994, Proc. SPIE, 2198, 362

Vogt, S. S., Radovan, M., Kibrick, R., et al. 2014, PASP, 126, 359

Winn, J. N. 2010, in Exoplanets, Exoplanet Transits and Occultations, ed. S. Seager (Tucson, AZ: Univ. Arizona Press), 55

Wright, E. L., Eisenhardt, P. R. M., Mainzer, A. K., et al. 2010, AJ, 140,1868 